Document downloaded from:

http://hdl.handle.net/10251/141956

This paper must be cited as:

Sans, M.; Selga, J.; Vélez, P.; Bonache, J.; Rodríguez Pérez, AM.; Boria Esbert, VE.; Martin Antonlin, F. (2018). Compact Wideband Balanced Bandpass Filters With Very Broad Common-Mode and Differential-Mode Stopbands. IEEE Transactions on Microwave Theory and Techniques. 66(2):737-750. https://doi.org/10.1109/TMTT.2017.2785246

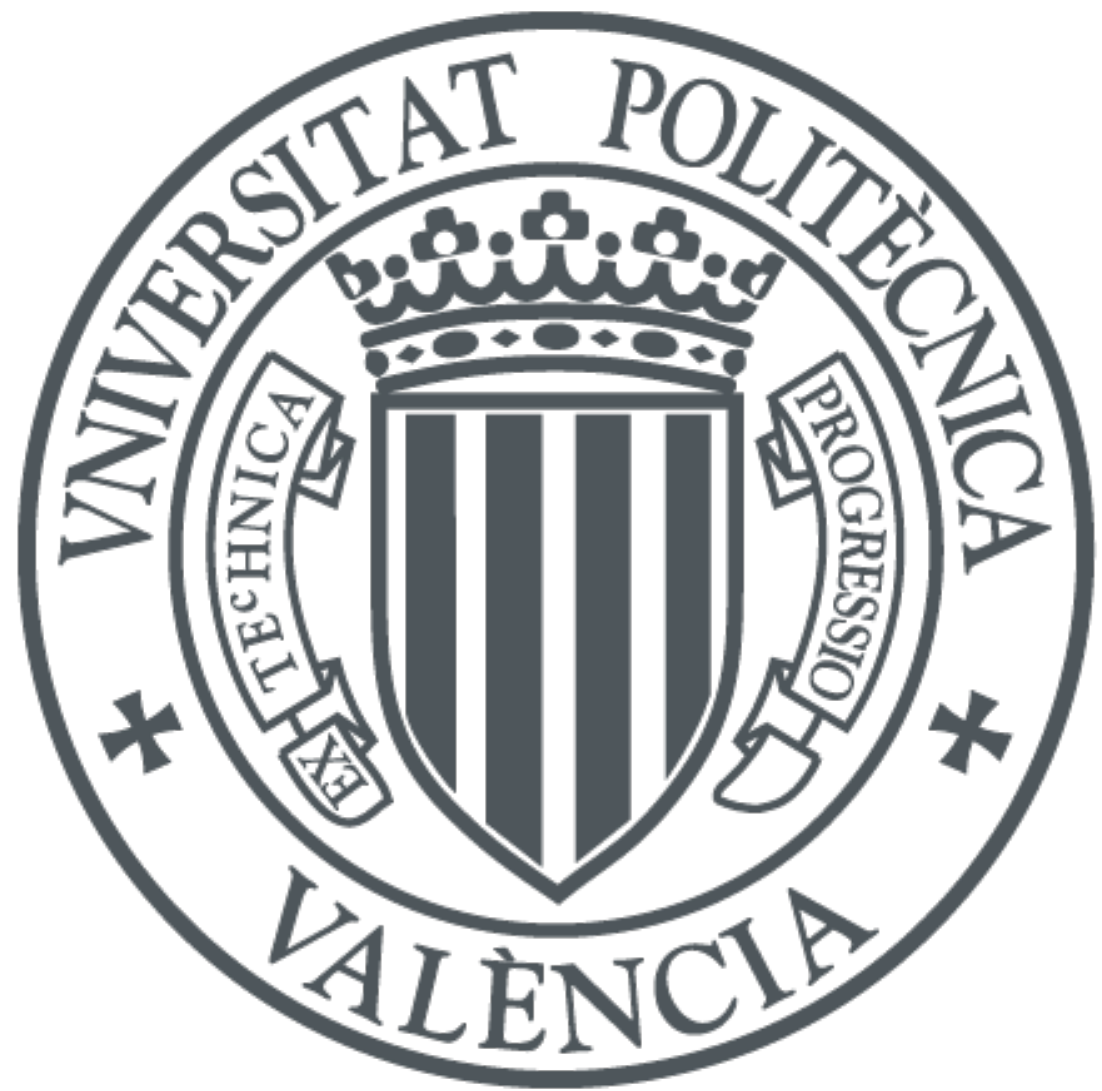

The final publication is available at

https://doi.org/10.1109/TMTT.2017.2785246

Copyright Institute of Electrical and Electronics Engineers

Additional Information 


\title{
Compact Wideband Balanced Bandpass Filters With Very Broad Common-Mode and Differential-Mode Stopbands
}

\author{
Marc Sans, Jordi Selga, Member, IEEE, Paris Vélez, Member, IEEE, Jordi Bonache, Member, IEEE, Ana \\ Rodríguez, Member, IEEE, Vicente E. Boria, Senior Member, IEEE, and Ferran Martín, Fellow, IEEE
}

\begin{abstract}
Compact balanced bandpass filters based on a combination of multisection mirrored stepped-impedance resonators and interdigital capacitors are presented in this paper. The considered filter topology is useful to achieve wide bandwidths for the differential mode, with broad stop bands for that mode, as well as very efficient common-mode suppression. By conveniently adjusting the transmission zeros for both operation modes, the differential- and common-mode stopbands can be extended up to significantly high frequencies. Filter size and this differential- and common-mode stopband performance are the main relevant characteristics of the proposed balanced filters. The potential of the approach is illustrated by the design of a prototype order-5 balanced bandpass filter, with central frequency $f_{0}=1.8 \mathrm{GHz}, 48 \%$ fractional bandwidth (corresponding to $55.4 \%-3-\mathrm{dB}$ bandwidth), and 0.04-dB ripple level. The filter is automatically synthesized by means of an aggressive space-mapping software tool, specifically developed, and two (pre- and post-) optimization algorithms, necessary to determine the transmission-zero frequencies. The designed filter is as small as $0.48 \lambda_{g} \times 0.51 \lambda_{g}$, where $\lambda_{g}$ is the guided wavelength at the central filter frequency, and the differential-mode stopband extends up to at least $6.5 \mathrm{GHz}$ with more than 22 -dB rejection. The common-mode suppression is better than $28 \mathrm{~dB}$ from dc up to at least $6.5 \mathrm{GHz}$.
\end{abstract}

Index Terms-Balanced bandpass filters, common-mode noise suppression, interdigital capacitors, microstrip technology, space mapping, stepped-impedance resonators (SIRs).

\section{INTRODUCTION}

B ALANCED (or differential mode) microwave filters have attracted the interest of the microwave community in recent years. The reason is the increasing demand of differential circuits and systems, due to the high immunity to

Manuscript received July 28, 2017. This work was supported in part by MINECO-Spain under Project TEC2013-40600-R, Project TEC2016-75650-R, and Project TEC2016-75934-C4-1-R, in part by the Generalitat de Catalunya under Project 2014SGR-157, in part by the Institució Catalana de Recerca i Estudis Avançats (who awarded F. Martín), and in part by by FEDER funds. This paper is an expanded version from the IEEE MTT-S International Conference on Numerical Electromagnetic Modeling and Optimization for RF, Microwave, and Terahertz Applications, Seville, Spain, May 17-19, 2017. (Corresponding author: Jordi Selga.)

M. Sans, J. Selga, P. Vélez, J. Bonache, and F. Martín are with the GEMMA/CIMITEC, Departament d'Enginyeria Electrònica, Universitat Autònoma de Barcelona, 08193 Bellaterra, Spain (e-mail: jordi.selga@uab.cat; Ferran.Martin@uab.es).

A. Rodríguez and V. E. Boria are with the Departamento de Comunicaciones-iTEAM, Universitat Politècnica de València, 46022 Valencia Spain (e-mail: vboria@dcom.upv.es). noise, electromagnetic interference, and crosstalk of differential signals [1], [2]. Ideally, balanced filters should be able to efficiently suppress the (parasitic) common mode over the widest possible band, and simultaneously exhibit good performance for the mode of interest (differential mode), including low insertion loss and high return loss in the passband, high filter selectivity, and a broad stopband (spurious cancellation). Filter size, design simplicity, and cost are also important aspects.

Solutions to suppress the common mode based on cascaded common-mode filters have been reported [3]-[5]. However, this strategy is not the optimum solution in terms of filter size. For this reason, common-mode suppressed balanced filters are mostly implemented through approaches that inherently reject the common mode. Following these approaches, narrow-band [6]-[11], dual-band [4], [12]-[20], wideband [21]-[38], and ultrawideband [39]-[47] balanced bandpass filters have recently reported.

This paper is focused on the design and synthesis of differential-mode bandpass filters, first reported in [36], based on a combination of multisection mirrored stepped-impendence resonators (SIRs) and interdigital capacitors. The proposed topology is useful for the implementation of wide-band responses for the differential mode. Due to the presence of multiple (controllable) transmission zeros for the differen-tial and common modes and to the blocking effect of the series connected interdigital capacitors at low frequencies, the common mode is efficiently suppressed from dc up to high frequencies (covering the differential-mode passband and beyond). Good performance in the passband and broad stopband for the differential mode is also achieved with the proposed topology. Moreover, filter size is reduced as compared to other similar filters based on quarter wavelength admittance inverters [34], since these filters are implemented only with semilumped (i.e., electrically small) components. The proposed filters are fully planar, can be fabricated in commercially available microwave substrates, use only two metallic levels, and do not use metallic vias. Hence, they represent a low-cost solution for wideband balanced filters. Finally, the layout of the filters is obtained following an automated scheme, by means of a developed software tool that implements an aggressive space-mapping (ASM) optimization and synthesis algorithm. 
As compared to the work presented in [36], in this paper, we provide a detailed analysis of the circuit model, including the equations that provide the reactive element values from filter specifications. We discuss in detail the developed ASM algorithm, necessary to automatically generate the filter layout, as well as the pre- and postoptimization algorithms necessary to determine the positions of the transmission-zero frequencies. Finally, we report an order-5 common-mode suppressed balanced filter, and we dedicate a section to compare the size and performance of such filter with other balanced filters (exhibiting comparable differential-mode bandwidth) available in the literature.

This paper is organized as follows. The filter topology and the equivalent circuit model are presented in Section II. Such section includes the analysis of the circuit model, necessary to achieve the required differential-mode filter response. The determination of the transmission-zero frequencies (for both the differential and common modes), necessary to achieve broadband common-mode suppression, and efficient rejection of the differential mode in the differential-mode stopband, is discussed in Section III. Section IV is devoted to the design and synthesis tool, based on ASM (as mentioned before), able to provide the filter layout following an unattended scheme. An illustrative design example is reported in Section V, specifically a fifth-order wideband balanced filter. In Section VI, a comparative analysis with other wideband balanced filters is carried out. Finally, the main conclusions are highlighted in Section VII.

\section{Filter Topology, Lumped Element EQUiVALENT CIRCUIT, AND ANALYSIS}

The typical topology of the wideband balanced bandpass filters under consideration is depicted in Fig. 1 [36]. Such topology corresponds to a fifth-order filter, but it can be generalized to an arbitrary order by simply adding or removing filter stages. All filter elements can be described by means of simple reactive elements in the frequency region of interest. This means that the filter is electrically small by nature, since it is composed by semilumped elements, rather than by distributed components.

The first-, third-, and fifth-filter stages are transverse mirrored SIRs made of seven alternating sections with high/low characteristic impedance. The second- and fourth-filter stages are made of differential pairs of interdigital capacitors cascaded between inductive (narrow) strips. The elliptical windows etched in the ground plane have the purpose of reducing the parasitic capacitances of the interdigital capacitors and increasing the per unit length inductance of the inductive strips. With this topology, wide bandwidths for the differential mode can be achieved, since it is possible to obtain low reactance slope for the series resonators (interdigital capacitors in series with the inductive strips), and low susceptance slope for the shunt resonators (made by a combination of mirrored SIR elements, as will be later shown). It is interesting to mention that the presence of series connected interdigital capacitors blocks signal transmission at low frequencies for both the differential and common modes. This is particularly useful for the suppression of the common mode at low frequencies.

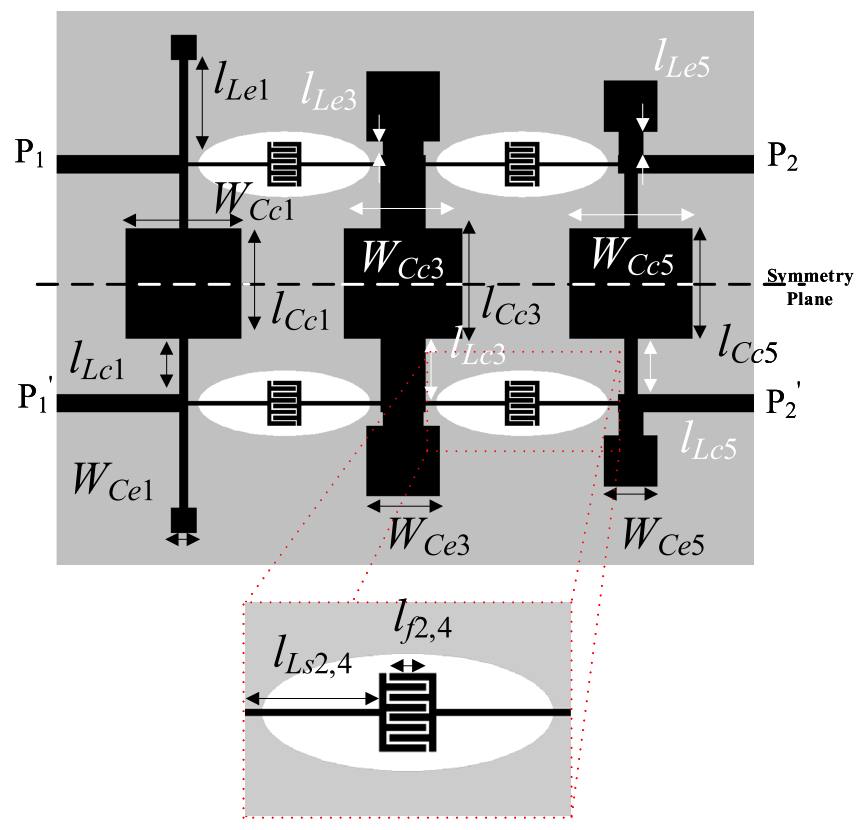

Fig. 1. Topology of the proposed balanced bandpass filters. The upper metal level and ground plane are depicted in black and gray, respectively. Relevant dimensions are indicated.

The lumped element equivalent circuit of the filter is shown in Fig. 2(a). The inductances and capacitances designated as $L_{e, i}$ and $C_{e, i}$ account for the external inductive strips (with length $l_{L e, i}$ ) and capacitive patches (with width $W_{C e, i}$ ), respectively, of the mirrored SIRs, whereas $L_{c, i}$ and $C_{c, i}$ describe the central inductive strips (with length $l_{L c, i}$ ) and central patches (with width $W_{C c, i}$ ), respectively. The interdigital capacitances are called $C_{s, i}$, whereas $L_{s, i}$ corresponds to the inductive strips cascaded to the interdigital capacitors. The capacitance $C_{\mathrm{par}}$ is a parasitic capacitance, necessary to adequately describe the interdigital capacitors and narrow inductive strips adjacent to them. Note, however, that $C_{\mathrm{par}}$ is part of the shunt resonators of the preceding and following filter stages, and are, therefore, relevant for the determination of the reactive elements of such stages, as will be later discussed. The subindex $i$ added to the reactive elements of the circuit model indicates the corresponding filter stage.

The circuit models for the differential and common modes are depicted in Fig. 2(b) and (c), respectively. Note that the unique difference between the differential- and commonmode circuits is the absence of the capacitance $C_{c, i}$ in the former circuit. The central patch capacitances are grounded for the differential mode and do not play any role for that mode. However, such capacitances, in combination with the inductances of the central strips, $L_{c, i}$, provide transmission zeros for the common mode, useful for the suppression of this mode. It is interesting to mention that the external resonators, $L_{e, i}-C_{e, i}$, provide transmission zeros for both the differential and common modes. Such transmission zeros are useful to further enhance the common-mode stopband bandwidth, to improve the filter selectivity for the differential mode, and to obtain a broad stopband for this mode above the differentialmode passband.

In the design of the filter, the first step is the determination of the elements of the circuit model from filter specifications, 


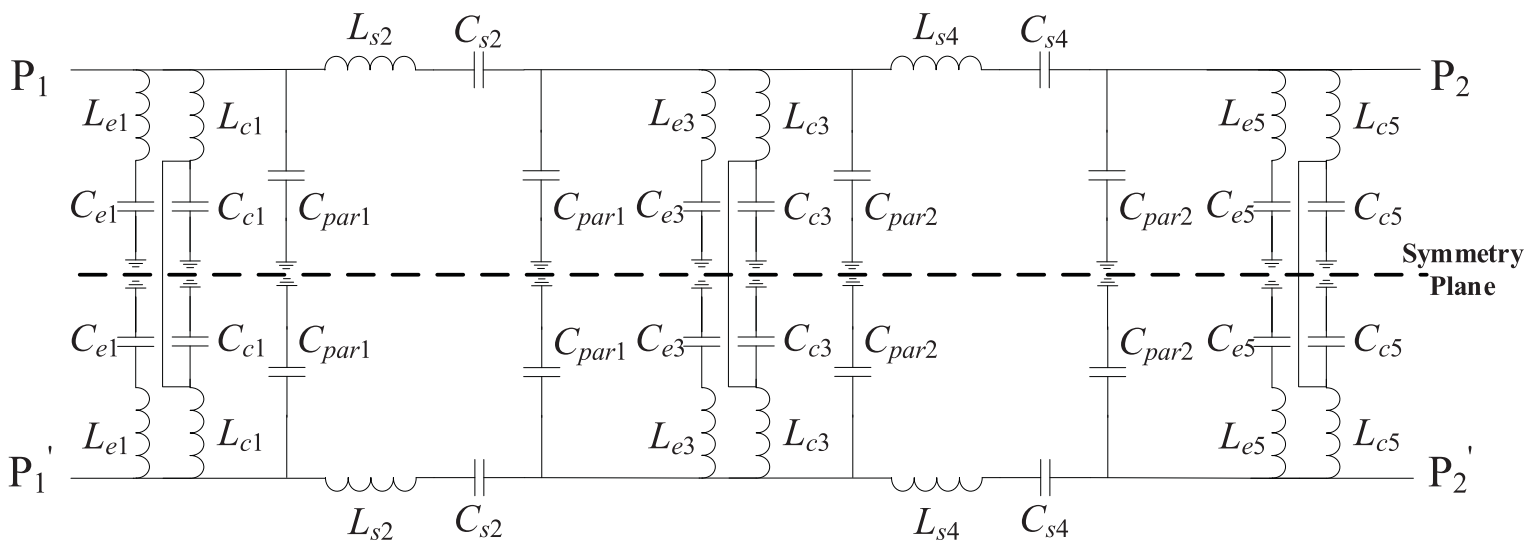

(a)

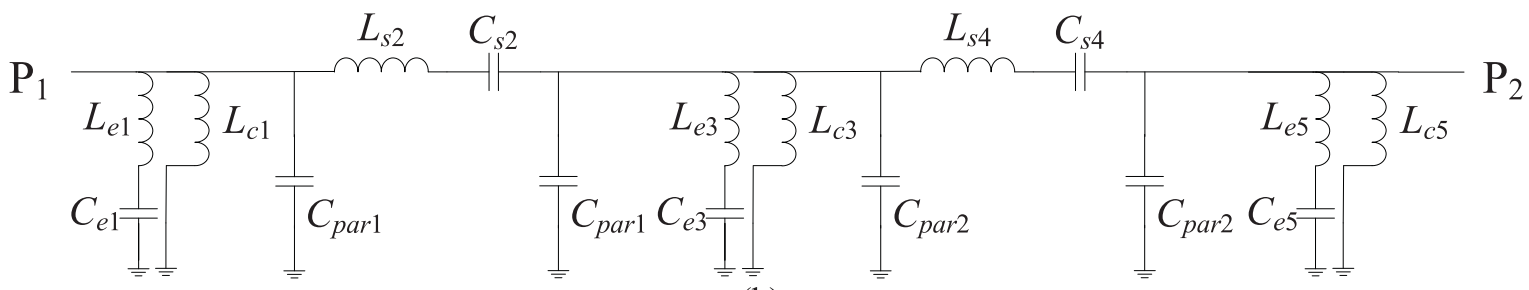

(b)

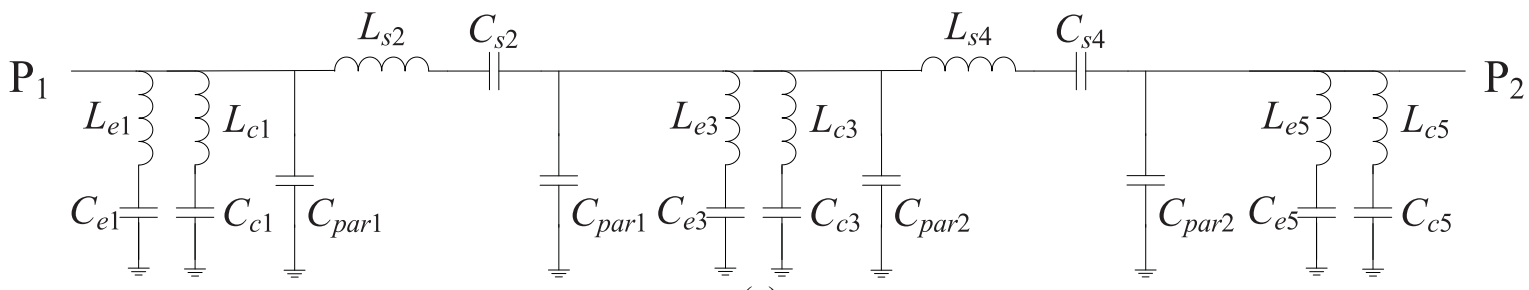

(c)

Fig. 2. (a) Lumped element equivalent circuit model of the filter of Fig. 1(a), and equivalent circuits for the (b) differential and (c) common modes.

and by the position of the differential- and common-mode transmission zeros. Once such elements are known, the second step is the determination of the filter layout, to be discussed in Section IV. In the circuit model, the capacitances $C_{\mathrm{par}}$ are the single parasitic elements. Such capacitances are due to the series stages (interdigital capacitors and narrow inductive strips), but, as mentioned before, they are part of the shunt stages. The capacitances $C_{\mathrm{par}}$ are not easily controllable parameters, but they can be easily inferred once the layouts of the series stages are known. Therefore, in the design flowchart, it is first necessary to determine the series elements, $L_{s, i}$ and $C_{s, i}$, and then synthesize the layouts of the interdigital capacitors and inductive strips. From such layouts, the values of capacitances $C_{\mathrm{par}}$ can be derived. The element values $L_{s, i}$ and $C_{s, i}$ can be easily determined from filter specifications (central frequency, bandwidth, filter order, and ripple level), according to standard filter design techniques [48], [49]. Let us assume that the capacitances $C_{\mathrm{par}}$ have been inferred from the layouts of the series stages (to be discussed in next section). The other reactive elements of the shunt branches are determined as follows. For the differential mode, the reactance of the shunt branches is given by

$$
\chi_{s, \text { diff }}=\frac{\left(\omega^{2} L_{e} C_{e}-1\right) L_{c}}{\omega^{3} C_{\mathrm{par}} L_{e} C_{e} L_{c}-\omega\left(C_{e} L_{c}+C_{e} L_{e}+C_{\mathrm{par}} L_{c}\right)}
$$

where the subindex $i$ has been omitted for simplicity. The three unknowns that appear in (1), $L_{c}, L_{e}$, and $C_{e}$, are given by the position of the transmission zeros for the differential mode

$$
f_{z, d d}=\frac{1}{2 \pi \sqrt{L_{e} C_{e}}}
$$

by the filter central frequency, where the shunt reactance for the differential mode, $\chi_{s \text {, diff, }}$ opens

$$
f_{0}=\frac{1}{2 \pi} \sqrt{\frac{C_{e} L_{c}+C_{e} L_{e}+C_{\mathrm{par}} L_{c}}{C_{\mathrm{par}} L_{e} C_{e} L_{c}}}
$$

and by the susceptance slope

$b=\left.\frac{\omega_{0}}{2} \frac{d B}{d \omega}\right|_{\omega_{0}}=\frac{\omega_{0} C_{\mathrm{par}}}{2}+\frac{1}{2 L_{c} \omega_{0}}+\frac{C_{e} \omega_{0}\left(1+L_{e} C_{e} \omega_{0}^{2}\right)}{2\left(1-L_{e} C_{e} \omega_{0}^{2}\right)^{2}}$

where $\omega_{0}=2 \pi f_{0}$ is the central angular frequency. The capacitances of the central patches, $C_{c, i}$, are determined from the positions of the common-mode transmission zeros, i.e.,

$$
f_{z, c c}=\frac{1}{2 \pi \sqrt{L_{c} C_{c}}} \text {. }
$$

Thus, from the solution of (2)-(5), the reactive element values of the shunt branches of the filter are inferred. 
Concerning the transmission zeros, it should be noted that there are as many differential-mode transmission zeros as shunt branches, or $(N+1) / 2$, where $N$ is the filter order (it is considered that the filter order is odd). The differentialmode transmission zeros are also common-mode transmission zeros. This helps to broaden the common-mode rejection $(\mathrm{CMR})$ bandwidth. However, $(N+1) / 2$ additional common-mode transmission zeros, due to the central patch capacitors, are available to suppress the common mode in the frequency region corresponding to the differential-mode passband.

\section{Optimization OF THE TRANSMission-ZERO FREQUENCIES}

In this paper, rather than a single (degenerated) commonmode transmission zero, the zeros for that operation mode are distributed in order to achieve good CMR within the differential-mode passband and beyond. With these transmission zeros, and those for the differential mode, necessarily set above the passband of interest (but also active for the common mode), the common mode can be significantly rejected beyond the differential-mode passband, as will be shown later.

The first (lower) differential-mode transmission zero must be positioned not very far from the upper differential-mode cutoff frequency. By this means, filter selectivity at the upper transition band is substantially improved. However, we should avoid locating the first differential-mode transmission zero too close to the upper cutoff frequency, in order to prevent the degradation of the differential-mode return loss in the upper part of the passband. According to the previous comments, a tradeoff is necessary.

In practice, it is difficult to a priori predict the positions of the transmission zeros (for the differential and common modes) necessary to optimize the differential and CMR bands. The reason is that filter design is focused on achieving a certain differential-mode response (dictated by specifications). However, the common-mode response (not subjected to specifications) typically exhibits transmission peaks. The position of such peaks depends on the transmission-zero frequencies, but not straightforwardly. Therefore, an iterative algorithm for the optimization of the transmission-zero frequencies (for both the differential and common modes) has been developed (pre-optimization algorithm). To illustrate how this algorithm works, let us consider a specific (conducting) filter example, corresponding to the following specifications: order $N=5$, quasi-Chebyshev bandpass response with central frequency $f_{0}=1.8 \mathrm{GHz}$, fractional bandwidth $\mathrm{FBW}=48 \%$ (or $55.4 \%$ -3-dB fractional bandwidth), and ripple level $L_{\mathrm{ar}}=0.04 \mathrm{~dB}$. (These specifications correspond to the filter to be designed later on.)

In a first step, the location of all the transmission zeros (differential and common modes) is set to an arbitrary single frequency, significantly above the upper cutoff frequency of the differential-mode passband, e.g., $2.5 f_{0}$. From filter specifications, we can infer the value of $L_{S}$ and $C_{S}$, corresponding to the second and fourth stages, using well-known formulas [49]. Since the elements of the shunt branches depend on $C_{\text {par }}$ [see expression (1)], it is necessary to first synthesize the layout of the series branches, according to the ASM algorithm to be specified later, and, from it, determine the value of $C_{\mathrm{par}}$. Once this parasitic capacitance is known $\left(C_{\text {par }}=0.445 \mathrm{pF}\right.$, as indicated later), (2)-(5) can be used to determine $L_{e}, L_{c}, C_{e}$, and $C_{c}$ for the first-, third-, and fifth-filter stages, and the response of the circuit (differential and common modes) at the schematic level can be inferred [Fig. 3(a)]. From this response, we can identify the common-mode transmission maxima at frequencies 1.942 and $2.047 \mathrm{GHz}$. (Note that these frequencies can be automatically obtained by means of a simple algorithm able to detect transmission maxima.)

In the second step, we reallocate two of the common-mode transmission zeros in the transmission peak frequencies (for that mode) and set the additional common-mode transmission zero to $1.5 f_{0}(2.7 \mathrm{GHz})$. The differential-mode transmission zeros are kept unaltered in this second step. Thus, the transmission zeros are: $f_{z, c c}=1.942 \mathrm{GHz}, 1.5 f_{0}, 2.047 \mathrm{GHz}$ and $f_{z, d d}=2.5 f_{0}, 2.5 f_{0}, 2.5 f_{0}$. The new response is the one depicted in Fig. 3(b), where the common-mode transmission peaks are found to be at $1.829,1.915$, and $3.103 \mathrm{GHz}$.

According to the previous common-mode transmission peaks, in a new (third) iteration, the transmission zeros are set to $f_{z, c c}=1.829 \mathrm{GHz}, 1.5 f_{0}, 1.915 \mathrm{GHz}$ and $f_{z, d d}=$ $3.103 \mathrm{GHz}, 2.5 f_{0}, 2.5 f_{0}$. Note that the third-common-mode transmission peak (at $3.103 \mathrm{GHz}$ ) is canceled by means of the first-differential-mode transmission zero. The new filter response is found to be the one depicted in Fig. 3(c), where it can be appreciated that the common-mode transmission peaks are significantly attenuated. Nevertheless, in this iterative algorithm, the objective is to obtain common-mode transmission peaks within the differential-mode passband with a level below $-25 \mathrm{~dB}$. The common-mode transmission peaks are found to be located at 1.811 and $1.895 \mathrm{GHz}$. Hence, the new set of transmission zeros in the fourth iteration is set to $f_{z, c c}=1.811 \mathrm{GHz}, 1.5 f_{0}, 1.895 \mathrm{GHz}$ and $f_{z, d d}=$ $3.103 \mathrm{GHz}, 2.5 f_{0}, 2.5 f_{0}$. The new response is depicted in Fig. 3(d), where there are still two peaks above $-25 \mathrm{~dB}$ at frequencies 1.808 and $1.892 \mathrm{GHz}$. Hence, the novel set of transmission zeros is $f_{z, c c}=1.808 \mathrm{GHz}, 1.5 f_{0}, 1.892 \mathrm{GHz}$ and $f_{z, d d}=3.103 \mathrm{GHz}, 2.5 f_{0}, 2.5 f_{0}$. With these transmission zeros, the filter response [Fig. 3(e)] exhibits CMR above $25 \mathrm{~dB}$ up to at least $2.5 f_{0}$. Therefore, the algorithm stops at this fifth iteration, and the common-mode and differential-mode transmission zeros are located at $f_{z, c c}=1.808 \mathrm{GHz}, 2.7 \mathrm{GHz}$, $1.892 \mathrm{GHz}$ and $f_{z, d d}=3.103 \mathrm{GHz}, 4.5 \mathrm{GHz}, 4.5 \mathrm{GHz}$, respectively.

The comparison between the obtained differential-mode response (circuit level) and the theoretical (ideal) Chebyshev response is depicted in Fig. 4 (the circuit elements of the final circuit are indicated). Although somehow degraded, the return loss in the passband is acceptable on account of the improved filter selectivity beyond the upper cutoff frequency (upper transition band).

\section{Filter Synthesis Through ASM}

Layout generation from the lumped element values is carried out following an ASM scheme [50]-[52]. Specific ASM algorithms for the series resonators (interdigital capacitors 


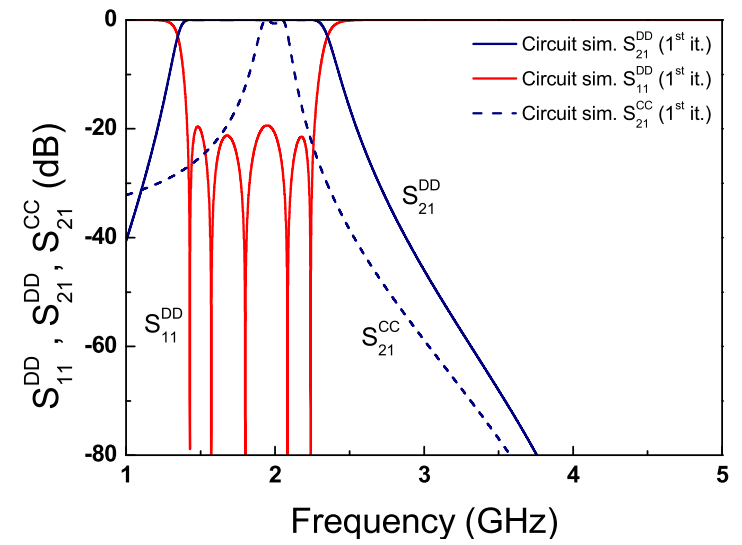

(a)

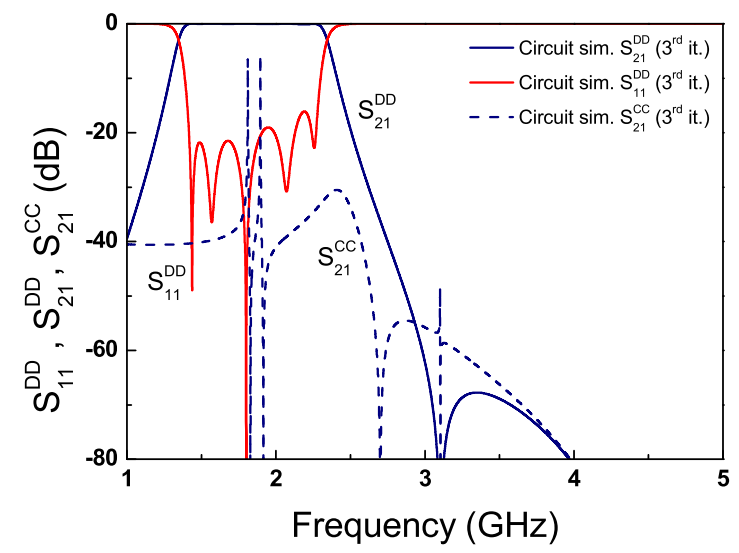

(c)

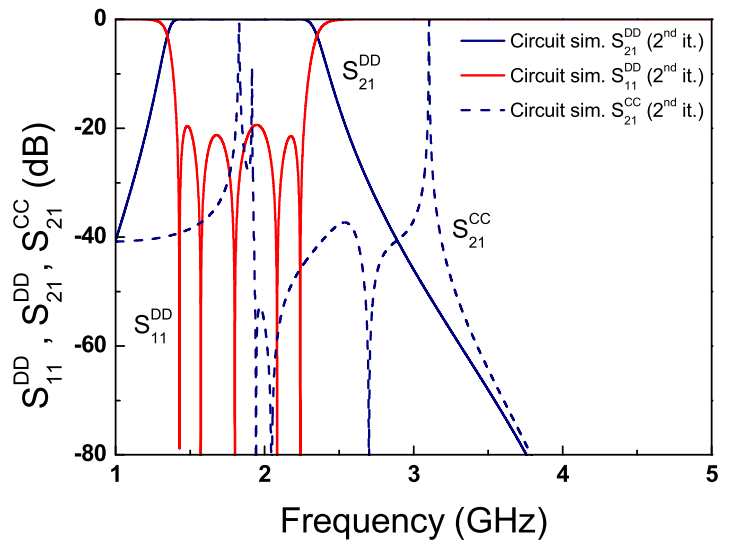

(b)

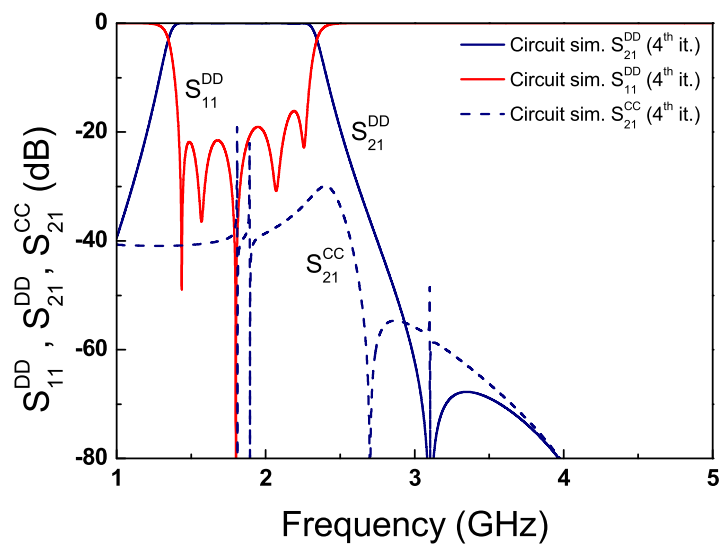

(d)

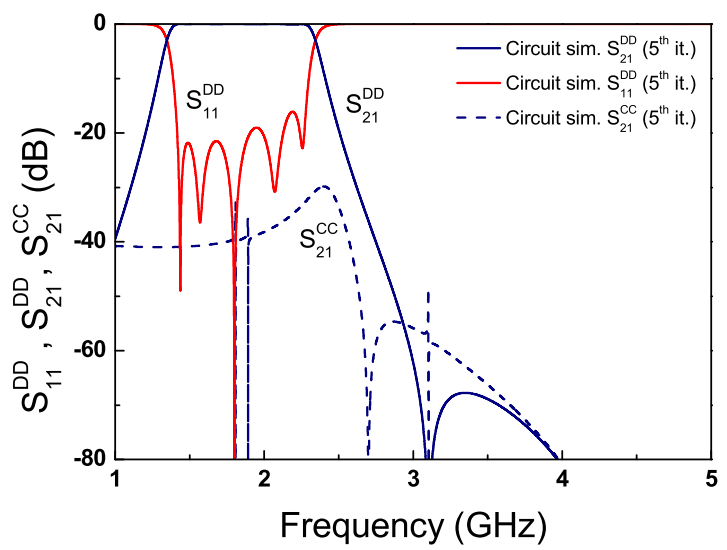

(e)

Fig. 3. Evolution of the differential- and common-mode responses (circuit level) obtained by means of the iterative algorithm used for the optimization of the transmission-zero positions. (For the common mode, only the insertion loss is depicted.) (a) First, (b) second, (c) third, (d) fourth, and (e) fifth iterations.

cascaded between narrow inductive strips) and for the mirrored SIRs have been developed.

\section{A. Synthesis of the Series Resonators}

For the series resonators, the variables in the optimization space are $L_{s, i}$ and $C_{s, i}$, whereas the variables in the validation space are the length of the inductive strip, $l_{L s}$, and the length of the fingers, $l_{f}$. The separation between fingers is set to $0.2 \mathrm{~mm}$. The width of the fingers and inductive strips is set to $0.4 \mathrm{~mm}$. To initiate the ASM algorithm, seeding values for $l_{L s}$ and $l_{f}$ are required. These values are inferred from well-known expressions providing the inductance of a narrow strip and the capacitance of an interdigital capacitor [48]. Then, the element values $L_{S, i}$ and $C_{S, i}$ are obtained through parameter extraction, and the first estimation of the Jacobian matrix $\mathbf{B}$ is obtained using a finite difference scheme [51]. Specifically, $l_{L s}$ and $l_{f}$ are perturbed, and the effects of these perturbations on the variation of $L_{s, i}$ and $C_{s, i}$ are expressed as derivatives in matrix form. From the extracted values of $L_{s, i}$ and $C_{s, i}$, the first error function is obtained

$$
\mathbf{f}\left(\mathbf{x}_{\mathbf{f}}\right)=\mathbf{P}\left(\mathbf{x}_{\mathbf{f}}\right)-\mathbf{x}_{\mathbf{c}}^{*}
$$




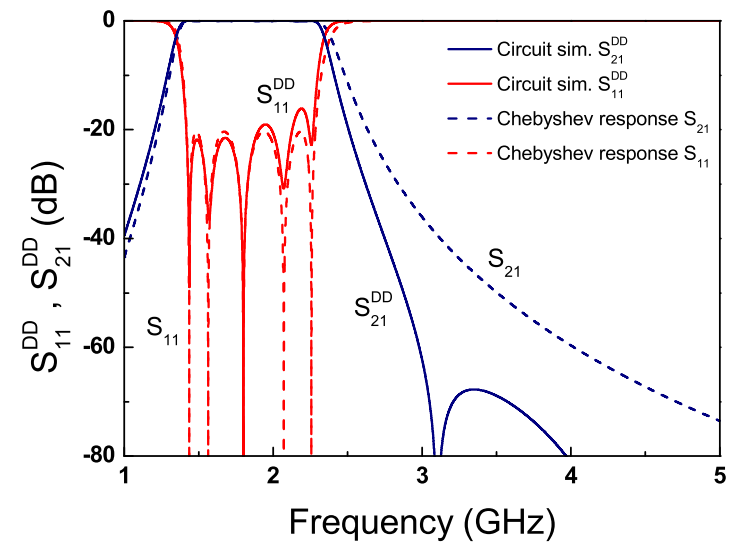

Fig. 4. Comparison between the ideal Chebyshev response subjected to the filter specifications and the differential-mode response of the filter (circuit level) resulting from the iterative algorithm used to determine the location of the transmission zeros. For stages second and fourth, $L_{s}=12.622 \mathrm{nH}$ and $C_{s}=0.619 \mathrm{pF}$ and $C_{\mathrm{par}}=0.445 \mathrm{pF}$. For the shunt branches, the circuit elements are $L_{e, 1}=1.938 \mathrm{nH}, L_{c, 1}=3.139 \mathrm{nH}, C_{e, 1}=1.358 \mathrm{pF}$, and $C_{c, 1}=2.468 \mathrm{pF}$ (first stage), $L_{e, 3}=0.311 \mathrm{nH}, L_{c, 3}=1.378 \mathrm{nH}$, $C_{e, 3}=4.020 \mathrm{pF}$, and $C_{c, 3}=2.522 \mathrm{pF}$ (third stage), and $L_{e, 5}=0.575 \mathrm{nH}$, $L_{c, 5}=2.576 \mathrm{nH}, C_{e, 5}=2.176 \mathrm{pF}$, and $C_{c, 5}=2.747 \mathrm{pF}$ (fifth stage).

where $\mathbf{x}_{\mathbf{c}}^{*}$ is the vector in the optimization space containing the objective (target) values, $\mathbf{x}_{\mathbf{f}}$ is the vector in the validation space, with the seeding values for $l_{L s}$ and $l_{f}$ in the first iteration, and $\mathbf{P}\left(\mathbf{x}_{\mathbf{f}}\right)$ is the vector in the optimization space resulting from parameter extraction from $\mathbf{x}_{\mathbf{f}}$. If we call $\mathbf{x}_{\mathbf{f}}^{(j)}$, the $j$ th approximation to the solution in the validation space, and $\mathbf{f}^{(j)}$ the corresponding error function, the next vector of the validation space is obtained according to

$$
\mathbf{x}_{\mathbf{f}}^{(j+1)}=\mathbf{x}_{\mathbf{f}}^{(j)}+\mathbf{h}^{(j)}
$$

where $\mathbf{h}^{(j)}$ is given by

$$
\mathbf{h}^{(j)}=-\left(\mathbf{B}^{(j)}\right)^{-1} \mathbf{f}^{(j)}
$$

and $\mathbf{B}^{(j)}$ is an approach to the Jacobian matrix, updated according to the Broyden formula [51]

$$
\mathbf{B}^{(j+1)}=\mathbf{B}^{(j)}+\frac{\mathbf{f}^{(j+1)} \mathbf{h}^{(j) T}}{\mathbf{h}^{(j) T} \mathbf{h}^{(j)}} .
$$

Convergence is considered to be achieved once the norm of the error function, defined as follows:

$$
\left\|f_{\text {norm }}\right\|=\sqrt{\left(1-\frac{L_{s}}{L_{s}^{*}}\right)^{2}+\left(1-\frac{C_{s}}{C_{s}^{*}}\right)^{2}}
$$

is smaller than a certain predefined value. Using this ASM scheme, the layouts of the series resonators are inferred. Once these layouts are known, the values of $C_{\mathrm{par}}$ for each series stage are easily obtained.

As an illustrative example, let us consider the synthesis of series resonators with $\mathbf{x}_{\mathbf{c}}^{*}=L_{s}^{*}, C_{s}^{*}=12.622 \mathrm{nH}, 0.619 \mathrm{pF}$, corresponding to stages second and fourth of the filter with specifications indicated in Section III (which are also the specifications of the order-5 balanced filter to be synthesized in next section as an example). The seeding values of the variables in the validation space are found to be $\mathbf{x}_{\mathbf{f}}^{(1)}=l_{L s}$, $l_{f}=9.399 \mathrm{~mm}, 4.112 \mathrm{~mm}$, and the reactive element values

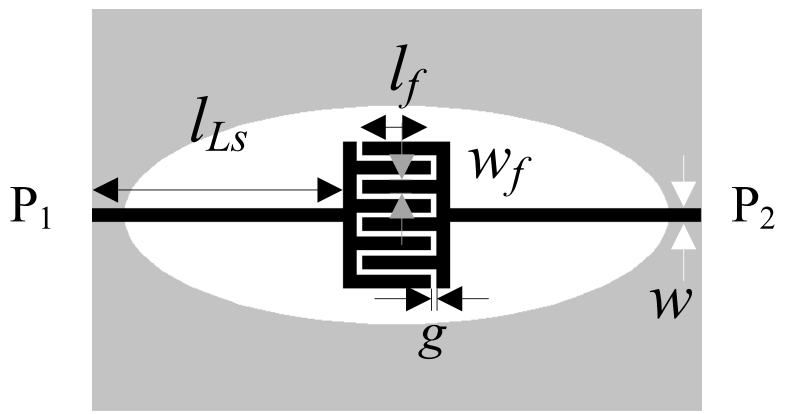

Fig. 5. Generated layout of the series resonator for the designed filter. Dimensions are (in millimeter): $w=0.4, l_{L s}=7.868, g=0.2, l_{f}=2.176$, and $w_{f}=0.4$.

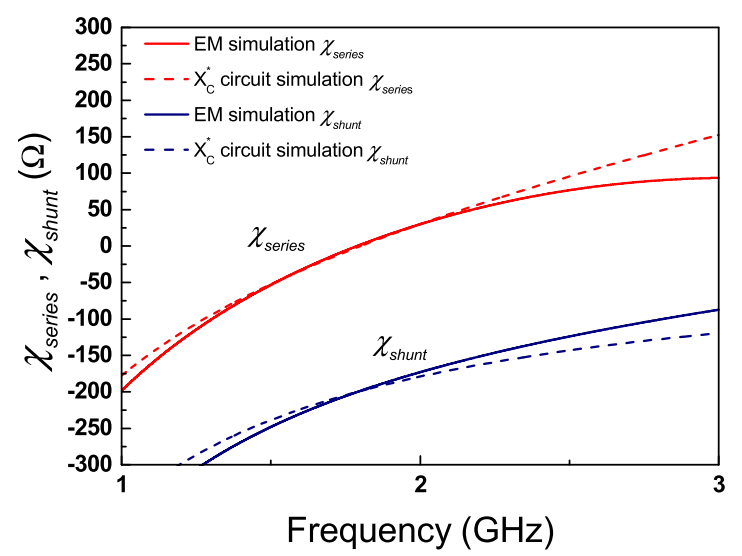

Fig. 6. Series and shunt reactance for the structure depicted in Fig. 5.

inferred through parameter extraction are found to be $\mathbf{x}_{\mathbf{c}}^{(1)}=$ $\mathbf{P}\left(\mathbf{x}_{\mathbf{f}}^{(1)}\right)=15.547 \mathrm{nH}, 1.026 \mathrm{pF}$. The considered substrate is Rogers RO4003C with dielectric constant $\varepsilon_{r}=3.55$, thickness $h=0.813 \mathrm{~mm}$, and loss tangent $\tan \delta=0.0021$. The specific parameter extraction is as simple as obtaining the series and shunt reactance of the equivalent $\pi$-circuit from the electromagnetic simulation of the layout (the Keysight momentum is used). The series elements, $L_{s}$ and $C_{s}$, are obtained from the resonance frequency and from the reactance slope. The shunt reactance provides the capacitance $C_{\text {par }}$ (it can be merely obtained from the slope of the susceptance). However, note that $C_{\mathrm{par}}$ is not used during the iteration process. As discussed in Section III, this capacitance is necessary to determine the elements of the shunt branches (mirrored SIRs), and it is fundamental in the optimization of the transmission-zero positions.

Using the ASM scheme explained before, convergence, with an error smaller than $1.7 \%$, is achieved after just 1 iteration. The final vectors in the optimization and validation spaces are $\mathbf{x}_{\mathbf{c}}^{(2)}=12.587 \mathrm{nH}, 0.629 \mathrm{pF}$, and $\mathbf{x}_{\mathbf{f}}^{(2)}=7.868 \mathrm{~mm}$, $2.176 \mathrm{~mm}$, respectively. The resulting parasitic capacitance is found to be $C_{\mathrm{par}}=0.445 \mathrm{pF}$. Fig. 5 depicts the synthesized layout, whereas the series and shunt reactances (inferred from electromagnetic simulation) are shown in Fig. 6. For comparison purposes, the target reactance (series reactance corresponding to $\mathbf{x}_{\mathbf{c}}^{*}$ ) and the reactance of $C_{\mathrm{par}}$ are also shown in Fig. 6. As can be seen, the agreement is good in the 
TABLE I

WIDTHS OF THE INDUCTIVE STRIPS OF THE MIRRORED SIRS

\begin{tabular}{|c|c|}
\hline $\boldsymbol{L}_{\boldsymbol{e}}, \boldsymbol{L}_{\boldsymbol{c}}(\mathrm{nH})$ & $\boldsymbol{W}_{\boldsymbol{L} \boldsymbol{e}}, \boldsymbol{W}_{\boldsymbol{L} \boldsymbol{c}}(\mathrm{mm})$ \\
\hline$>1.6$ & 0.8 \\
\hline$[1,1.6)$ & 1.6 \\
\hline$[0.5,1)$ & 2.4 \\
\hline$[0.35,0.5)$ & 3.2 \\
\hline$<0.35$ & 4 \\
\hline
\end{tabular}

region of interest, pointing out the validity of the employed ASM method.

\section{B. Synthesis of the Mirrored SIRs}

For the mirrored SIRs, the procedure is similar to the one reported in [34], but with significant differences. Such differences are due to the fact that in the filters reported in [34], based on mirrored SIRs coupled through admittance inverters, the SIRs were all identical, except the central patch (in order to achieve multiple transmission zeros for the common mode). The filter response in [34] was controlled by the impedance of the inverters, different in each stage. Therefore, the width of the pair of transmission-line sections (inverters) was a variable in the optimization process. However, the transverse distance between these lines was kept constant in [34] thanks to the identical admittance of the shunt (SIR) resonators for the differential mode. Keeping this distance invariable is convenient for design purposes. In this paper, the shunt resonators are not identical since the reactive elements of the SIRs are determined by the admittance slope and transmission zeros (besides the filter central frequency). Hence, the variables in the validation space (geometrical variables) will be different for the various SIRs in order to keep unaltered the transverse distance between the pair of lines along the filter. Conversely, the variables in the optimization space are in all cases $L_{e}, L_{c}, C_{e}$, and $C_{c}$.

Let us now explain in detail, the ASM algorithm applied to the synthesis of the mirrored SIRs. The first one (first stage) is optimized as follows: the widths of the inductive strips $W_{L c}$ and $W_{L e}$ are set to different values depending on the inductance value. Specifically, these widths are determined according to Table I.

Once these widths are fixed, the variables in the validation space are chosen to be the lengths of these inductances, $l_{L c}$ and $l_{L e}$, and the lengths of the capacitive patches, $l_{C c}$ and $l_{C e}$ (the widths of such patches are chosen to be identical to the lengths, i.e., $W_{C c}=l_{C c}$ and $W_{C e}=l_{C e}$, corresponding to a square geometry). Once the four geometrical variables that must be optimized have been decided, we proceed similar to the synthesis of the series resonant elements. From wellknown formulas that provide the inductance of a narrow strip and the capacitance of a patch, the seeding value of the geometrical variable is determined. After that, the reactive element parameters, $L_{e}, L_{c}, C_{e}$, and $C_{c}$, are extracted. The first Jacobian $(4 \times 4)$ matrix is obtained by perturbing the geometrical variables, and the first error function is obtained according to (6), where $\mathbf{x}_{\mathbf{c}}^{*}$ and $\mathbf{x}_{\mathbf{f}}$ are the ASM variables of the first mirrored SIR. The iterative process is then carried out using (7)-(9) until convergence is achieved.

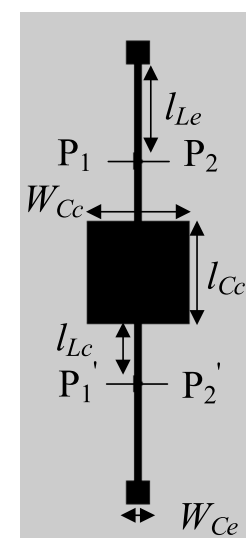

Fig. 7. Dimensions are (in millimeters): $W_{C e}=3.543, l_{L e}=6.404$, $W_{C c}=l_{C c}=10.785$, and $l_{L c}=5.668$.

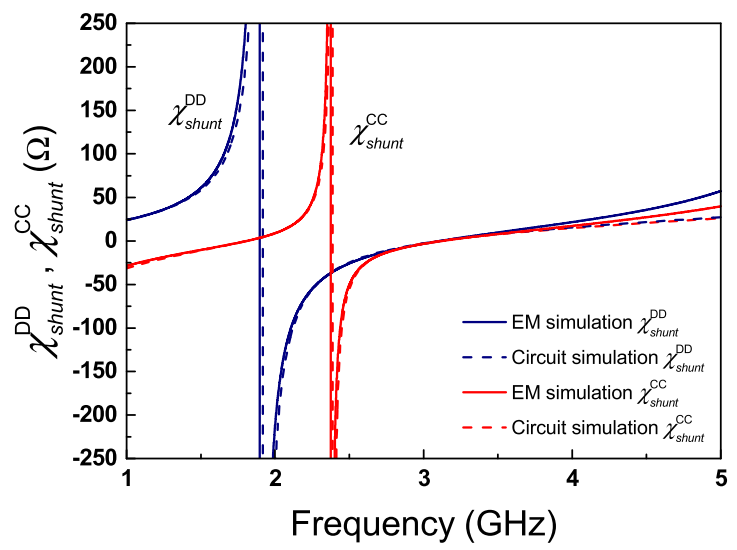

Fig. 8. Differential- and common-mode reactances for the mirrored SIR of Fig. 7.

As an illustrative example, let us consider the synthesis of a mirrored SIR with the following reactive element values (corresponding to the first SIR of the order-5 filter to be presented in next section): $L_{e}=1.938 \mathrm{nH}, L_{c}=3.139$ $\mathrm{nH}, C_{e}=1.358 \mathrm{pF}$, and $C_{c}=2.468 \mathrm{pF}$. The final vectors in the optimization and validation spaces (obtained after six iterations with an error smaller than $0.94 \%$ ) are found to be $\mathbf{x}_{\mathbf{c}}^{(6)}=L_{e}, L_{c}, C_{e}, C_{c}=1.935 \mathrm{nH}, 3.157 \mathrm{nH}, 1.354 \mathrm{pF}$, $2.452 \mathrm{pF}$, and $\mathbf{x}_{\mathbf{f}}^{(6)}=l_{L e}, l_{L c}, l_{C e}, l_{C c}=6.404 \mathrm{~mm}, 5.668 \mathrm{~mm}$, $3.543 \mathrm{~mm}, 10.785 \mathrm{~mm}$. The corresponding layout is depicted in Fig. 7, whereas the reactances for the differential and common modes inferred from electromagnetic simulations are depicted in Fig. 8. Such reactances are compared to those of the circuit model, and the agreement is very good, as can be appreciated in Fig. 8.

For the other mirrored SIRs, the lengths of the central patch and central inductive strip are set to the corresponding values of the first mirrored SIR, i.e., $l_{C c, i}=l_{C c, 1}$ and $l_{L c, i}=l_{L c, 1}$. By this means, the distance between terminals in the differential ports of the mirrored SIRs is identical in all the stages. Thus, the optimization variables in the validation space for such SIRs (with $i>1$ ) are: $l_{L e, i}$ (the width of this inductance set using Table I), $W_{L c, i}, W_{C c, i}$, and $l_{C e, i}$ (considering $W_{C e, i}=l_{C e, i}$ in order to obtain a square geometry for the external patches). 
TABLE II

GeOMETRIC PARAMETERS OF THE MiRRORED SIRs

\begin{tabular}{|c|c|c|c|c|c|c|c|c|}
\hline $\boldsymbol{i}$ & $\boldsymbol{l}_{\boldsymbol{L c}}$ & $\boldsymbol{l}_{\boldsymbol{C c}}$ & $\boldsymbol{l}_{\boldsymbol{L e}}$ & $\boldsymbol{l}_{\boldsymbol{C e}}$ & $\boldsymbol{W}_{\boldsymbol{L c}}$ & $\boldsymbol{W}_{\boldsymbol{C c}}$ & $\boldsymbol{W}_{\boldsymbol{L e}}$ & $\boldsymbol{W}_{\boldsymbol{C}}$ \\
\hline 1 & 5.668 & 10.785 & 6.404 & 3.543 & 0.8 & 10.785 & 0.8 & 3.543 \\
\hline 3 & 5.668 & 10.785 & 1.471 & 7.247 & 4.203 & 12.007 & 4 & 7.247 \\
\hline 5 & 5.668 & 10.785 & 2.457 & 5.270 & 1.215 & 11.858 & 2.4 & 5.270 \\
\hline
\end{tabular}

All dimensions in $\mathrm{mm}$

One important difference between the synthesis of these filters and those reported in [34] concerns the fact that in [34], the ASM algorithm was actually a two-step process. That is, due to the limited functionality (bandwidth) of the admittance inverters implemented by means of quarter wavelength transmission lines, it was first necessary that an ASM algorithm be able to provide the schematic necessary to compensate for the narrowing effects of the admittance inverters on the filter response (optimum schematic). This first ASM is not necessary for the design of the proposed wideband balanced filters since distributed components are not used. Therefore, by considering the filter topology reported here, a clear benefit in terms of design simplicity and quickness is obtained (nevertheless, an iterative, but very fast, algorithm for the optimization of the transmission-zero frequencies has been necessary, as discussed in Section III).

\section{Prototype Device Example}

To illustrate the potential of the proposed ASM approach, the filter with specifications indicated in Section III and with the transmission zeros (differential and common modes) allocated at the indicated positions is reported in this section. With such specifications, the series elements $L_{s}$ and $C_{s}$ (identical for stages $i=2$ and $i=4$ ), inferred from the low-pass filter prototype Chebyshev tables corresponding to the cited order and ripple, and from well-known impedance and response transformations [48], are those indicated in Section III. The value of $C_{\mathrm{par}}$ and the element values of the mirrored SIRs (found from the optimization of the transmission-zero frequencies) are also indicated in Section III (see Fig. 4).

Once these element values are known, layout generation for the series resonators and for each mirrored SIR is obtained by means of the ASM scheme explained in previous section (illustrated in detail for the series resonators and for the mirrored SIR of the first filter stage). The geometric values of the series resonators (interdigital capacitors and inductive strips) are given in Section IV-A, and those corresponding to the different mirrored SIRs are indicated in Table II.

The filter layout is obtained by cascading the synthesized mirrored SIRs and series resonators. The electromagnetic simulation (using Keysight Momentum), including losses, of the filter response for the differential and common modes is depicted in Fig. 9. Despite the fact that the circuit model of the proposed filters provides a reasonable response in the region of interest, it can be appreciated in Fig. 9 that the common mode resulting from lossy electromagnetic simulation (the response of interest) exhibits transmission peaks above $-20 \mathrm{~dB}$. The reason is that such peaks are very sensitive to the position of the transmission zeros (as seen in Section III), which may

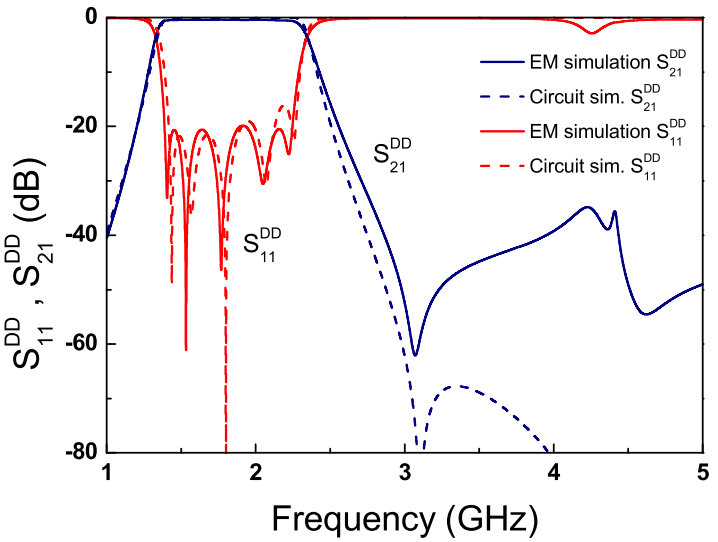

(a)

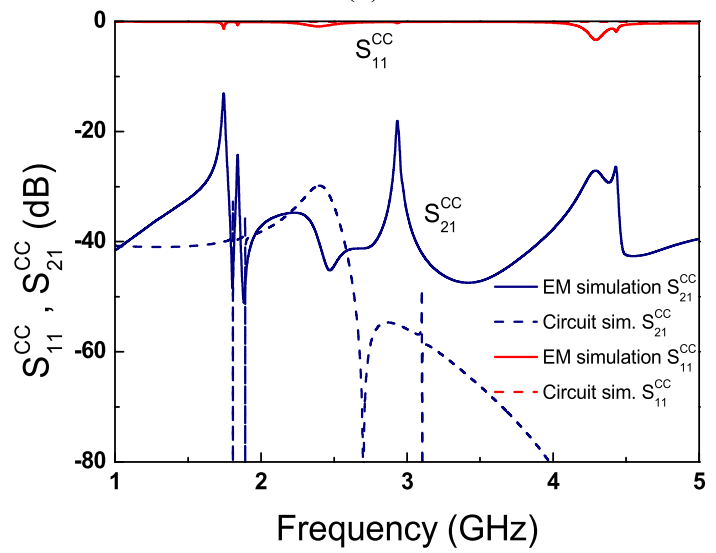

(b)

Fig. 9. Lossy electromagnetic and lossless circuit simulation of the designed balanced filter. (a) Differential-mode response. (b) Common-mode response.

experience some shift in the lossy electromagnetic simulation, as compared to the circuit response.

Since CMR is not satisfactory enough, readjustment of the transmission-zero positions is needed. So, we have developed a second (postoptimization) iterative algorithm similar to the one reported in Section III, but this time the responses are not the circuit simulations of the resulting equivalent circuits, but the electromagnetic simulations (including losses) of the filter after application of the ASM synthesis algorithm. Obviously, this is time consuming, but convergence is obtained after few iterations, provided the initial response (the one inferred from the first ASM-Fig. 9) already exhibits a CMR level better than the target $(25 \mathrm{~dB})$, except at certain frequencies. By applying this postoptimization iterative algorithm to the structure resulting from the first ASM, we have been able (after three iterations) to obtain a filter layout exhibiting a CMR level better than $-28 \mathrm{~dB}$. The reactive elements of the final filter circuit and the geometrical elements of the optimized layout are indicated in Tables III and IV, respectively. Note that $L_{S}, C_{S}, C_{\mathrm{par}}$, and the geometry of the series resonators is kept unaltered in this postoptimization iterative process, since the readjustment of the transmission zeros does not affect the series branches. The final layout of the filter is the one depicted in Fig. 1, and the photograph is shown in Fig. 10 (the filter has been fabricated by means of photo mask etching). 
TABLE III

ReACtive Element VAlues of the Optimized Filter

\begin{tabular}{|c|c|c|c|c|c|c|}
\hline $\boldsymbol{i}$ & $\boldsymbol{L}_{\boldsymbol{c}}(\mathbf{n H})$ & $\boldsymbol{C}_{\boldsymbol{c}}(\mathbf{p F})$ & $\boldsymbol{L}_{\boldsymbol{e}}(\mathbf{n H})$ & $\boldsymbol{C}_{\boldsymbol{e}}(\mathbf{p F})$ & $\boldsymbol{L}_{\boldsymbol{s}}(\mathbf{n H})$ & $\boldsymbol{C}_{\boldsymbol{s}}(\mathbf{p F})$ \\
\hline 1 & 3.303 & 2.639 & 2.456 & 1.199 & --- & --- \\
\hline 2,4 & --- & --- & --- & --- & 12.622 & 0.619 \\
\hline 3 & 1.378 & 2.522 & 0.311 & 4.020 & --- & --- \\
\hline 5 & 2.576 & 2.934 & 0.575 & 2.176 & --- & --- \\
\hline
\end{tabular}

TABLE IV

GeOMETRIC PARAMETERS OF THE MiRRORED SIRs FOR THE OPTIMIZED FILTER

\begin{tabular}{|c|c|c|c|c|c|c|c|c|}
\hline $\boldsymbol{i}$ & $\boldsymbol{l}_{\boldsymbol{L c}}$ & $\boldsymbol{l}_{\boldsymbol{C c}}$ & $\boldsymbol{l}_{\boldsymbol{L} \boldsymbol{e}}$ & $\boldsymbol{l}_{\boldsymbol{C e}}$ & $\boldsymbol{W}_{\boldsymbol{L c}}$ & $\boldsymbol{W}_{\boldsymbol{C c}}$ & $\boldsymbol{W}_{\boldsymbol{L e}}$ & $\boldsymbol{W}_{\boldsymbol{C e}}$ \\
\hline 1 & 5.754 & 11.401 & 9.9 & 2.587 & 0.8 & 11.401 & 0.8 & 2.587 \\
\hline 3 & 5.754 & 11.401 & 1.462 & 7.209 & 4.415 & 11.668 & 4 & 7.209 \\
\hline 5 & 5.754 & 11.401 & 2.462 & 5.259 & 1.272 & 12.148 & 2.4 & 5.259 \\
\hline
\end{tabular}

All dimensions in $\mathrm{mm}$

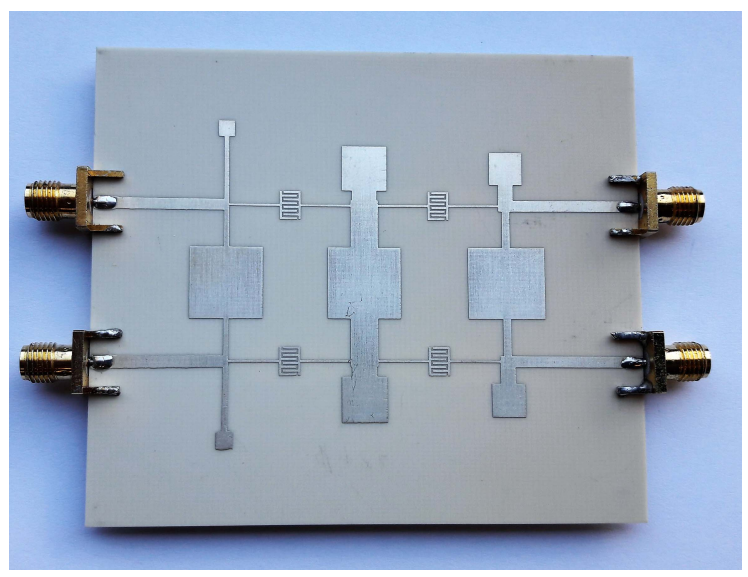

Fig. 10. Photograph of the fabricated balanced filter.

Figs. 11 and 12 depict the lossless and lossy, respectively, electromagnetic simulation of the final filter, including the differential- and common-mode responses. The circuit simulation is also included in Fig. 11. The very good agreement between the circuit and lossless electromagnetic simulation for the differential mode indicates the validity of the proposed filter model (discrepancies above $3 \mathrm{GHz}$ are due to the fact that the filter elements cannot be described by simple reactive elements). Obviously, such agreement is not so good for the common mode, due to the high sensitivity of this mode to the position of the transmission zeros (which has forced us to implement a postoptimization iterative algorithm). Nevertheless, an acceptable coincidence up to approximately $3 \mathrm{GHz}$, except by the level of the common-mode transmission peaks, can be observed as well. Note that, as we have mentioned before, the postoptimization algorithm is based on forcing the lossy electromagnetic response of the common mode to be below a certain value $(-25 \mathrm{~dB})$. Thus, the fact that in Fig. 11(b) such threshold level is not satisfied does not mean that the final filter layout is not optimized. Indeed, in view of Fig. 12(b), the common-mode response inferred from lossy electromagnetic simulation exhibits a rejection level better than $-28 \mathrm{~dB}$. Therefore, the target commonmode response is clearly satisfied with the synthesized filter layout.

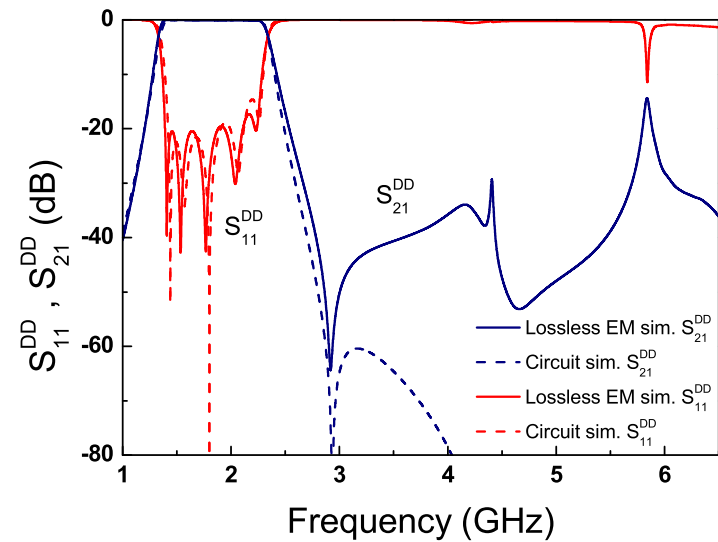

(a)

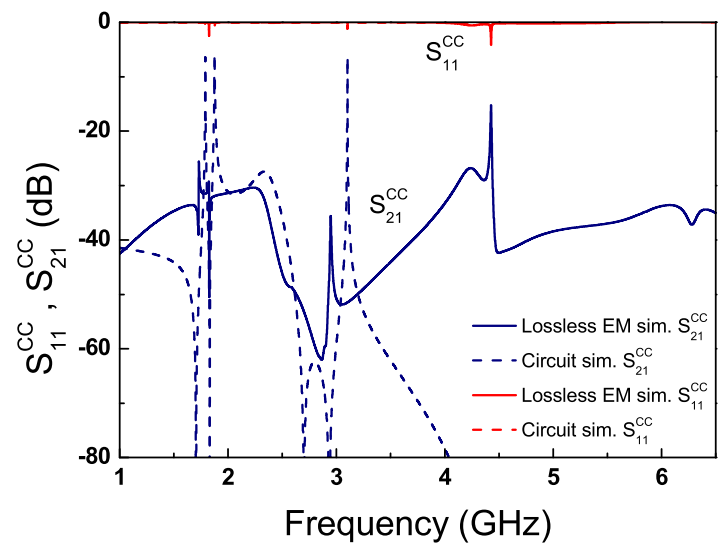

(b)

Fig. 11. Lossless electromagnetic and circuit simulation of the designed (optimized) balanced filter. (a) Differential mode. (b) Common mode.

The measured filter responses for the differential and common modes have been obtained by means of the Agilent PNA N5221A vector network analyzer. Such responses are included in Fig. 12 for a proper comparison with the lossy electromagnetic simulation. The agreement is good, although the measured in-band return loss for the differential mode is somehow degraded, as compared to the electromagnetic simulation. This is due to tolerances in the fabrication process. Note that interdigital capacitors have very small gap $(g=0.2 \mathrm{~mm})$, and this is a critical parameter. The sensitivity analysis of Fig. 13, showing the effects of slight variations in $g$ on the frequency response, confirms this point. Also, perfect symmetry is also difficult to achieve in practice, and there are two pairs of symmetric interdigital capacitors that make this aspect more critical. Nevertheless, the measured in-band return loss (differential mode) is better than $11.5 \mathrm{~dB}$. To obtain this measured value, we have forced an in-band ripple at design level (controllable form the element vales of the low-pass filter prototype) small enough $(0.04 \mathrm{~dB})$, having in mind the unavoidable degradation of the corresponding return loss due to losses, tolerances, and, in our case, due to the presence of differential-mode transmission zeros close the band of interest. Concerning the in-band insertion loss, it is better than $1 \mathrm{~dB}$. The differential-mode stopband exhibits a rejection level better than $35 \mathrm{~dB}$ up to $5.6 \mathrm{GHz}$ (i.e., slightly above $3 f_{0}$ ), but such 


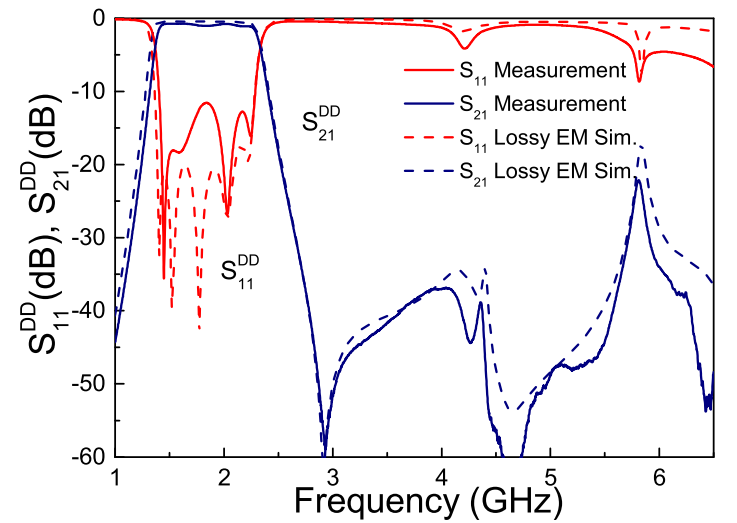

(a)

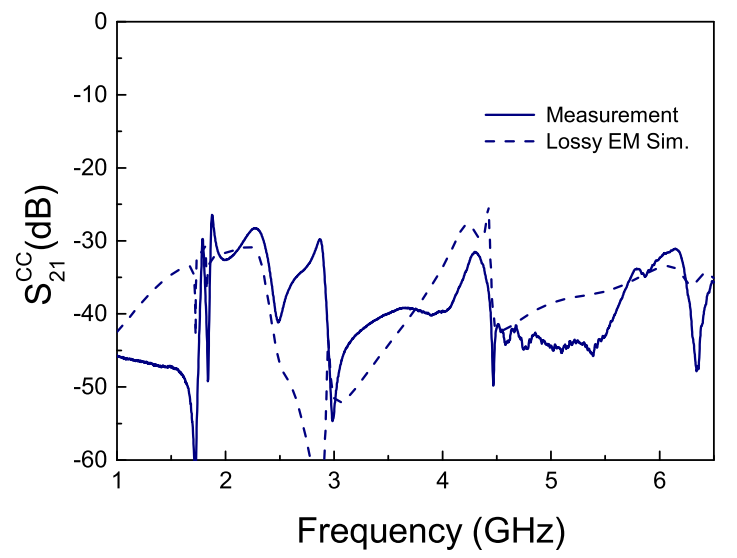

(b)

Fig. 12. Lossy electromagnetic simulation and measured response of the designed (optimized) and fabricated balanced filter. (a) Differential mode. (b) Common mode.

band extends up to at least $6.5 \mathrm{GHz}$ with rejection level better than $22 \mathrm{~dB}$. The common mode is suppressed with a rejection level better than $28 \mathrm{~dB}$ from dc up to at least $6.5 \mathrm{GHz}$. Finally, filter size is as small as $51.5 \mathrm{~mm} \times 48.2 \mathrm{~mm}$, corresponding to $0.51 \lambda_{g} \times 0.48 \lambda_{g}$ in terms of the wavelength at the filter central frequency $f_{0}$. This combination of size and filter performance is competitive, as will be discussed in the next section.

\section{COMPARISON TO OTHER WIDEBAND BALANCED FILTERS}

The reported filter is compared to other wideband balanced bandpass filters in Table V. Such table includes the differential-mode fractional bandwidth (DM-FBW), the central frequency $\left(f_{0}\right)$, the CMR level, including the frequency interval, the differential-mode upper stopband (DM-USB), indicating the stopband rejection level and frequency span, and the effective area, expressed in terms of the square wavelength (with wavelength calculated at $f_{0}$ ). Concerning the CMR, if it is below $-28 \mathrm{~dB}$ (the value achieved in the proposed filter), we have opted to indicate the frequency interval corresponding to this value for proper comparison. The exception is the filter in [29], where the reported common-mode insertion loss is limited to a narrow band and we cannot determine the frequency span corresponding to a CMR better than $-28 \mathrm{~dB}$.

In view of Table V, the filter of Fig. 10 exhibits very good CMR bandwidth. None of the filters reported in the references

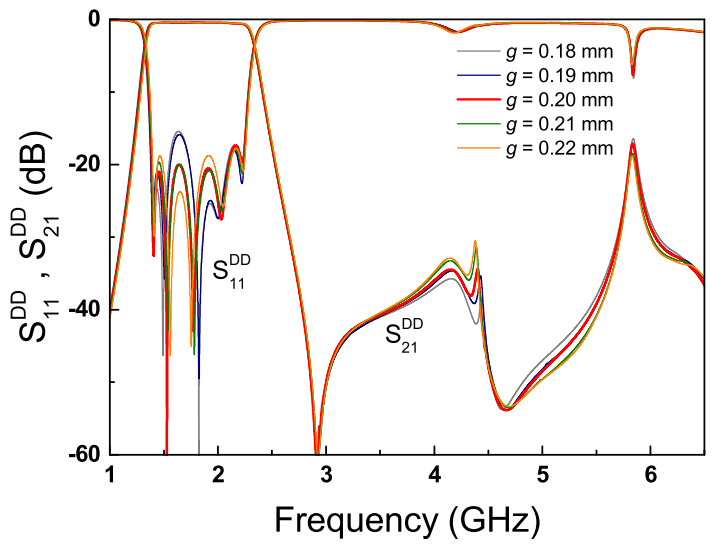

(a)

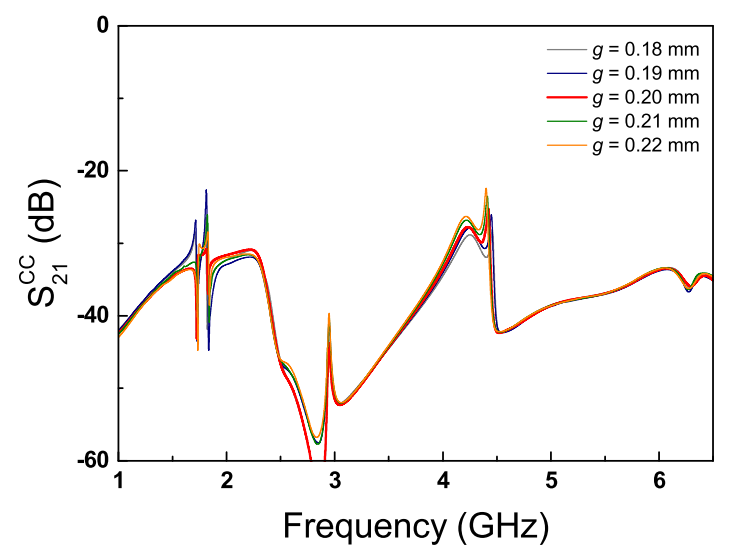

(b)

Fig. 13. Frequency responses of the proposed filter obtained through lossy electromagnetic simulation by varying the gap distance $g$ of the interdigital capacitors. (a) Differential mode. (b) Common mode. Variations are between 0.18 and $0.22 \mathrm{~mm}$ in steps of $0.01 \mathrm{~mm}$.

appearing in Table $\mathrm{V}$ exhibits a bandwidth extending from dc up to at least $3.6 f_{0}$ with such CMR $\left(\left|S_{21, c c}\right|<-28 \mathrm{~dB}\right)$. Indeed, in the filters with lower CMR, the CMR bandwidth is also narrower, as compared to our filter. Nevertheless, the filter of [32] is quite competitive in terms of commonmode suppression performance, since the CMR is better than $-27 \mathrm{~dB}$ from dc up to $2.8 f_{0}$. In some of the filters, e.g., those of [34], the maximum CMR at $f_{0}$ is very high (i.e., $\left|S_{21, c c}\right|=-65 \mathrm{~dB}$ and $\left|S_{21, c c}\right|=-50 \mathrm{~dB}$ for filters designated as $[34]^{a}$ and $[34]^{b}$ in Table V). However, in those filters, such huge rejection level is limited to a narrow bandwidth, as seen in Table V.

Concerning the differential-mode response, specifically the upper stopband (DM-USB), the most competitive filters are those of [23], [33], and [34] and the filter of Fig. 10. Specifically, the filter in [33] exhibits a very good combination of stopband bandwidth and rejection level $(-53 \mathrm{~dB})$, but the CMR bandwidth is very limited in this filter. It is worth mentioning that in the filter reported in this paper, there is a transmission zero located very close to the upper cutoff frequency (differential mode). The consequence is high filter selectivity in the upper transition band, with a roll off of roughly $-80 \mathrm{~dB} / \mathrm{GHz}$. Such high selectivity provides 
TABLE V

COMPARISON OF VARIOUS DIFFERENTIAL WIDEBAND BANDPASS FILTERS

\begin{tabular}{|c|c|c|c|c|c|}
\hline Ref. & $\begin{array}{c}\text { DM-FBW } \\
(-3 \mathrm{~dB})\end{array}$ & $\begin{array}{c}f_{0} \\
(\mathbf{G H z})\end{array}$ & $\begin{array}{c}\text { CMR }\left|S_{21, \mathrm{cc}}\right| \\
\text { (dB) }\end{array}$ & $\begin{array}{c}\text { DM-USB } \\
\left|S_{21, \mathrm{dd}}\right| \\
(\mathrm{dB})\end{array}$ & $\begin{array}{c}\text { Effective } \\
\text { area }\left(\lambda_{g}{ }^{2}\right) / \\
\text { order }\end{array}$ \\
\hline$[21]$ & $65 \%$ & 4.0 & $\begin{array}{c}<-20 \\
{\left[0.6 f_{0}, 1.4 f_{0}\right]}\end{array}$ & -- & $0.25 / 3$ \\
\hline$[22]$ & $50 \%$ & 4.1 & $\begin{array}{c}<-16 \\
{\left[0.3 f_{0}, 1.7 f_{0}\right]}\end{array}$ & $\begin{array}{c}<-18 \\
\left(>2.2 f_{0}\right)\end{array}$ & $0.25 / 3$ \\
\hline$[23]$ & $45 \%$ & 1.0 & $\begin{array}{c}<-25 \\
{\left[0,1.2 f_{0}\right]}\end{array}$ & $\begin{array}{l}<-40 \\
\left(2.7 f_{0}\right)\end{array}$ & $0.045 / 3$ \\
\hline$[25]$ & $43 \%$ & 2.4 & $\begin{array}{c}<-28 \\
{\left[0.6 f_{0}, 1.5 f_{0}\right]}\end{array}$ & $\begin{array}{l}<-36 \\
\left(2.1 f_{0}\right)\end{array}$ & $0.60 / 5$ \\
\hline [26] & $15 \%$ & 1.0 & $\begin{array}{c}<-25 \\
{\left[0,1.15 f_{0}\right]}\end{array}$ & $\begin{array}{l}<-20 \\
\left(1.8 f_{0}\right)\end{array}$ & $0.022 / 3$ \\
\hline$[27]$ & $70 \%$ & 3.0 & $\begin{array}{c}<-20 \\
{\left[0.8 f_{0}, 1.7 f_{0}\right]}\end{array}$ & --- & $0.52 /--$ \\
\hline [33] & $42 \%$ & 2.4 & $\begin{array}{c}<-28 \\
{\left[0.8 f_{0}, 1.4 f_{0}\right]}\end{array}$ & $\begin{array}{l}<-53 \\
\left(2.5 f_{0}\right)\end{array}$ & $0.37 / 5$ \\
\hline$[28]$ & $66.6 \%$ & 1.62 & $\begin{array}{l}<-14.5 \\
{\left[0,3.0 f_{0}\right]}\end{array}$ & $\begin{array}{l}<-30 \\
\left(2.5 f_{0}\right)\end{array}$ & $0.163 / 3$ \\
\hline [29] & $40 \%$ & 3.5 & $\begin{array}{c}<-40 \\
{\left[0.8 f_{0}, 1.2 f_{0}\right]}\end{array}$ & $\begin{array}{l}<-20 \\
\left(2.3 f_{0}\right)\end{array}$ & $0.25 / 3$ \\
\hline [30] & $53 \%$ & 3.0 & $\begin{array}{c}<-13 \\
{\left[0,3.0 f_{0}\right]}\end{array}$ & $\begin{array}{l}<-10 \\
\left(2.8 f_{0}\right)\end{array}$ & $0.175 /--$ \\
\hline [31] & $59.5 \%$ & 1.85 & $\begin{array}{c}<-28 \\
{\left[0.4 f_{0}, 1.6 f_{0}\right]}\end{array}$ & $\begin{array}{l}<-35 \\
\left(2.2 f_{0}\right)\end{array}$ & $0.39 / 3$ \\
\hline [32] & $56.7 \%$ & 3.0 & $\begin{array}{c}<-27 \\
{\left[0,>2.8 f_{0}\right]}\end{array}$ & $\begin{array}{c}<-31 \\
\left(>2.2 f_{0}\right)\end{array}$ & $0.20 / 3$ \\
\hline [24] & $79 \%$ & 3.0 & $\begin{array}{c}<-13 \\
{\left[0,2.7 f_{0}\right]}\end{array}$ & $\begin{array}{l}<-25 \\
\left(2.6 f_{0}\right)\end{array}$ & $0.192 / 5$ \\
\hline$[34]^{\mathrm{a}}$ & $43 \%$ & 2.4 & $\begin{array}{c}<-28 \\
{\left[0.8 f_{0}, 1.3 f_{0}\right]}\end{array}$ & $\begin{array}{c}<-44 \\
\left(>2.5 f_{0}\right)\end{array}$ & $0.36 / 5$ \\
\hline$[34]^{b}$ & $65 \%$ & 3.0 & $\begin{array}{c}<-28 \\
{\left[0.7 f_{0}, 1.5 f_{0}\right]}\end{array}$ & $\begin{array}{c}<-34 \\
\left(>2.5 f_{0}\right)\end{array}$ & $0.28 / 7$ \\
\hline [35] & $56 \%$ & 2.4 & $\begin{array}{c}<-15 \\
{\left[0,1.5 f_{0}\right]}\end{array}$ & --- & $0.17 / 3$ \\
\hline [36] & $43 \%$ & 1.5 & $\begin{array}{c}<-22 \\
{\left[0,2.5 f_{0}\right]}\end{array}$ & $\begin{array}{c}<-30 \\
\left(>1.2 f_{0}\right)\end{array}$ & $0.13 / 3$ \\
\hline [37] & $70 \%$ & 6.85 & $\begin{array}{c}(<-14.5) \\
{\left[0,>2.8 f_{0}\right]}\end{array}$ & $\begin{array}{c}<-30 \\
(>2.63 \\
\left.f_{0}\right) \\
\end{array}$ & $0.28 / 5$ \\
\hline [38] & 66 & 3.5 & $\begin{array}{c}<-6 \\
{\left[0.5 f_{0}, 1.4 f_{0}\right]}\end{array}$ & $\begin{array}{c}<-15 \\
\left(>2.3 f_{0}\right)\end{array}$ & $0.20 /--$ \\
\hline $\begin{array}{l}\text { This } \\
\text { work }\end{array}$ & $55.4 \%$ & 1.8 & $\begin{array}{c}<-28 \\
{\left[0,>3.6 f_{0}\right]}\end{array}$ & $\begin{array}{c}<-22 \\
\left(>3.6 f_{0}\right)\end{array}$ & $0.245 / 5$ \\
\hline
\end{tabular}

a differential-mode rejection level better than $-55 \mathrm{~dB}$ at $2.9 \mathrm{GHz}\left(1.61 f_{0}\right)$, and better than $-38 \mathrm{~dB}$ up to $5.6 \mathrm{GHz}$ $\left(3.11 f_{0}\right)$, and is a relevant aspect in the reported filter. Note that the filter of Fig. 10 exhibits the widest differential-mode stopband of all the filters included in Table V.

Concerning filter size, the filters of [23] and [26] are very competitive, but in both cases the CMR bandwidth is very limited, as compared to the filter reported in this paper. Note that these filters [23], [26] are order-3 filters, whereas the filter of Fig. 10 is a fifth-order filter. The third-order filters of [35] and [36] are also very small, but in terms of rejection bandwidth for both the differential and common modes, our filter is by far superior, as can be appreciated in the table. Note that, as mentioned in the introduction, the proposed filter (Fig. 10) is based on the topology of the order-3 filter of [36], from which it follows that the order-5 filter reported here should be electrically larger.

Finally, let us highlight the differences and advantages of the filter proposed in this paper as compared to recently reported similar differential filters proposed by Sans et al. [34], [35]. Indeed, the filter proposed in this paper combines the relevant advantageous aspects of the filters of [34] and [35] in the same structure, and at the same time overcomes the limiting aspects, resulting in the proposed new topology of Fig. 10. The result is a filter exhibiting a very good combination of filter size and performance, as compared to [34] and [35] (see Table V). The filters in [34] exhibit relatively good differential-mode stopband, but the common-mode stopband is limited since it does not start at dc. Moreover, these filters use distributed components (quarter-wavelength inverters), and this means that a first ASM to provide the optimum schematic (in order to compensate for the narrowing effect of thenonideal-transmission-line inverters) is required, as reported in [34]. In this paper, these limitations have been addressed with the proposed topology, thanks to the replacement of the transmission-line inverters with interdigital resonators. By these means, the common mode is rejected over a wide band starting at dc (due to the blocking effect of the series connected interdigital capacitances), and the first ASM used to obtain the optimum schematic is not necessary. Therefore, the introduction of interdigital capacitances represents a significant improvement as compared to the filters of [34], and eases filter design. Moreover, the proposed filter is also more compact, although the filter order is also different.

The main difference as compared to the filter reported in [35] concerns the mirrored SIRs. In the filters reported here, the mirrored SIRs extend out of the DM transmission lines (through the inductive strips with inductance $L_{e, i}$, not present in the filter of [35]). This provides transmission zeros for the differential mode (and also for the common mode), not present in [35], and by this means we achieve a good differential-mode upper stopband. The result is a differential filter with much superior performance.

An additional important and novel aspect proposed (and discussed) in this paper, with direct impact on filter design, is the pre- and postoptimization iterative algorithms considered for the optimization of the transmission-zero positions. Through these algorithms, not applied in [34] and [35], it has been possible to achieve a wide common-mode stopband with significant CMR (a crucial aspect in common-mode suppressed differential filters).

To summarize this section, the proposed filter exhibits a very good combination of size and differential- and common-mode stopband bandwidths. Moreover, filter synthesis has been carried out following an automated scheme. The approach combines an ASM algorithm (that provides filter layout from the elements of the circuit model) with pre- and postoptimization algorithms, necessary to optimize the position of the transmission zeros. (A fundamental aspect to achieve the reported differential- and common-mode stop bands.)

\section{CONCLUSION}

In conclusion, a wideband balanced bandpass filter with wide stopband for the differential mode, and common-mode suppression from dc up to frequencies well beyond the differential-mode passband, has been designed and fabricated. The filter has been implemented by cascading multisection 
mirrored SIRs and interdigital capacitors. The mirrored SIRs provide transmission zeros for the differential and common modes, useful to achieve very broad stopbands for both modes, keeping roughly unaltered the differential-mode passband. The interdigital capacitors are key elements to block the commonmode signals at low frequencies. Moreover, such capacitors, combined with narrow inductive strips, constitute the series resonators of the filter, resulting in a balanced filter fully implemented by means of semilumped (electrically small) elements.

Filter design has been carried out following an ASM optimization algorithm, where the geometry of the different mirrored (shunt connected) SIRs and series resonators has been inferred from the lumped elements of the equivalent circuit model. These elements, in turn, have been obtained from filter specifications and from the transmission-zero frequencies for both modes. Such transmission-zero positions have been determined by means of an iterative pre-optimization algorithm (at circuit level) that guarantees a minimum (specified) level of CMR within the differential-mode passband and beyond. Nevertheless, due to the high sensitivity of common-mode suppression to the transmission-zero frequencies, a postoptimization algorithm (at electromagnetic simulation level) to fine-tune the transmission-zero frequencies has been also developed.

As an illustrative example, the design and synthesis of a fifth-order balanced bandpass filter has been reported. The filter is small on account of the semilumped elements considered in its implementation, and filter performance for the differential mode is good, with insertion loss better than $1 \mathrm{~dB}$ in the passband and return loss higher than $11.5 \mathrm{~dB}$ in that band. The differential-mode stopband extends up to at least $6.5 \mathrm{GHz}\left(3.6 f_{0}\right.$ ), with more than $22-\mathrm{dB}$ rejection (and the rejection level is better than $38 \mathrm{~dB}$ up to $5.6 \mathrm{GHz}$, or $3.11 f_{0}$ ). Finally, the common mode is rejected with more than $28 \mathrm{~dB}$ from dc up to at least $6.5 \mathrm{GHz}\left(3.6 f_{0}\right)$. This combination of filter size and performance is very competitive. Although filter synthesis uses three specifically developed algorithms (an ASM-based algorithm preceded and followed by transmissionzero optimization algorithms), few iterations suffice to achieve convergence in all cases. This avoids excessive computing times, despite the fact that the latter transmission-zero optimization scheme uses the ASM algorithm at each iteration.

\section{REFERENCES}

[1] F. Martín, L. Zhu, F. Medina, and J. S. Hong, Balanced Microwave Filters. Hoboken, NJ, USA: Wiley, 2018.

[2] W. Feng, W. Che, and Q. Xue, "The proper balance: Overview of microstrip wideband balance circuits with wideband common mode suppression," IEEE Microw. Mag., vol. 16, no. 5, pp. 55-68, Jun. 2015.

[3] J. Naqui et al., "Common-mode suppression in microstrip differential lines by means of complementary split ring resonators: Theory and applications," IEEE Trans. Microw. Theory Techn., vol. 60, no. 10, pp. 3023-3034, Oct. 2012.

[4] A. Fernandez-Prieto et al., "Dual-band differential filter using broadband common-mode rejection artificial transmission line," Prog. Electromagn. Res., vol. 139, pp. 779-797, Apr. 2013.

[5] A. Fernández-Prieto et al., "Common-mode suppression for balanced bandpass filters in multilayer liquid crystal polymer technology," IET Microw., Antennas Propag., vol. 9, no. 12, pp. 1249-1253, Sep. 2015.
[6] C. H. Wu, C. H. Wang, and C. H. Chen, "Balanced coupled-resonator bandpass filters using multisection resonators for common-mode suppression and stopband extension," IEEE Trans. Microw. Theory Techn., vol. 55, no. 8, pp. 1756-1763, Aug. 2007.

[7] C. H. Wu, C. H. Wang, and C. H. Chen, "Stopband-extended balanced bandpass filter using coupled stepped-impedance resonators," IEEE Microw. Wireless Compon. Lett., vol. 17, no. 7, pp. 507-509, Jul. 2007.

[8] Y. Zhou, H.-W. Deng, and Y. Zhao, "Compact balanced-to-balanced microstrip diplexer with high isolation and common-mode suppression," IEEE Microw. Wireless Compon. Lett., vol. 24, no. 3, pp. 143-144, Mar. 2014.

[9] A. Fernández-Prieto, A. Lujambio, J. Martel, F. Medina, F. Mesa, and R. R. Boix, "Simple and compact balanced bandpass filters based on magnetically coupled resonators," IEEE Trans. Microw. Theory Techn., vol. 63, no. 6, pp. 1843-1853, Jun. 2015.

[10] A. Fernández-Prieto, J. Martel, F. Medina, F. Mesa, and R. R. Boix, "Compact balanced FSIR bandpass filter modified for enhancing common-mode suppression," IEEE Microw. Wireless Compon. Lett., vol. 25, no. 3, pp. 154-156, Mar. 2015.

[11] A. Fernández-Prieto, J. Bhatker, A. Lujambio, J. Martel, F. Medina, and R. R. Boix, "Balanced bandpass filter based on magnetically coupled coplanar waveguide folded-stepped impedance resonators," Electron. Lett., vol. 52, no. 14, pp. 1229-1231, Jul. 2016.

[12] C.-H. Lee, C.-I. G. Hsu, and C.-C. Hsu, "Balanced dual-band BPF with stub-loaded SIRs for common-mode suppression," IEEE Microw. Wireless Compon. Lett., vol. 20, no. 2, pp. 70-72, Feb. 2010.

[13] J. Shi and Q. Xue, "Balanced bandpass filters using center-loaded halfwavelength resonators," IEEE Trans. Microw. Theory Techn., vol. 58, no. 4, pp. 970-977, Apr. 2010.

[14] J. Shi and Q. Xue, "Dual-band and wide-stopband single-band balanced bandpass filters with high selectivity and common-mode suppression," IEEE Trans. Microw. Theory Techn., vol. 58, no. 8, pp. 2204-2212, Aug. 2010.

[15] J. Shi and Q. Xue, "Novel balanced dual-band bandpass filter using coupled stepped-impedance resonators," IEEE Microw. Wireless Compon. Lett., vol. 20, no. 1, pp. 19-21, Jan. 2010.

[16] Y.-H. Cho and S.-W. Yun, "Design of balanced dual-band bandpass filters using asymmetrical coupled lines," IEEE Trans. Microw. Theory Techn., vol. 61, no. 8, pp. 2814-2820, Aug. 2013.

[17] X. Xu, J. Wang, G. Zhang, and J. Chen, "Design of balanced dual-band bandpass filter based on substrate integrated waveguide," Electron. Lett., vol. 49, no. 20, pp. 1278-1280, Sep. 2013.

[18] F. Wei, Y. J. Guo, P. Y. Qin, and X. W. Shi, "Compact balanced dualand tri-band bandpass filters based on stub loaded resonators," IEEE Microw. Wireless Compon. Lett., vol. 25, no. 2, pp. 76-78, Feb. 2015.

[19] Y. Shen, H. Wang, W. Kang, and W. Wu, "Dual-band SIW differential bandpass filter with improved common-mode suppression," IEEE Microw. Wireless Compon. Lett., vol. 25, no. 2, pp. 100-102, Feb. 2015.

[20] F. Bagci, A. Fernández-Prieto, A. Lujambio, J. Martel, J. Bernal, and F. Medina, "Compact balanced dual-band bandpass filter based on modified coupled-embedded resonators," IEEE Microw. Wireless Compon. Lett., vol. 27, no. 1, pp. 31-33, Jan. 2017.

[21] T. B. Lim and L. Zhu, "A differential-mode wideband bandpass filter on microstrip line for UWB application," IEEE Microw. Wireless Compon. Lett., vol. 19, no. 10, pp. 632-634, Oct. 2009.

[22] T. B. Lim and L. Zhu, "Highly selective differential-mode wideband bandpass filter for UWB application," IEEE Microw. Wireless Compon. Lett., vol. 21, no. 3, pp. 133-135, Mar. 2011.

[23] P. Vélez et al., "Differential bandpass filter with common-mode suppression based on open split ring resonators and open complementary split ring resonators," IEEE Microw. Wireless Compon. Lett., vol. 23, no. 1, pp. 22-24, Jan. 2013.

[24] W. Feng, W. Che, Y. Ma, and Q. Xue, "Compact wideband differential bandpass filters using half-wavelength ring resonator," IEEE Microw. Wireless Compon. Lett., vol. 23, no. 2, pp. 81-83, Feb. 2013.

[25] P. Vélez et al., "Differential bandpass filters with common-mode suppression based on stepped impedance resonators (SIRs)," in IEEE MTT-S Int. Microw. Symp. Dig., Seattle, WA, USA, Jun. 2013, pp. 1-4.

[26] A. K. Horestani, M. Durá-Sindreu, J. Naqui, C. Fumeaux, and F. Martín, "S-shaped complementary split ring resonators and their application to compact differential bandpass filters with common-mode suppression," IEEE Microw. Wireless Compon. Lett., vol. 24, no. 3, pp. 149-151, Mar. 2014.

[27] X.-H. Wang and H. Zhang, "Novel balanced wideband filters using microstrip coupled lines," Microw. Opt. Technol. Lett., vol. 56, no. 5, pp. 1139-1141, 2014 
[28] L. Li, J. Bao, J.-J. Du, and Y.-M. Wang, "Compact differential wideband bandpass filters with wide common-mode suppression," IEEE Microw. Wireless Compon. Lett., vol. 24, no. 3, pp. 164-166, Mar. 2014.

[29] H. Wang, L. M. Gao, K. W. Tam, W. Kang, and W. Wu, "A wideband differential BPF with multiple differential- and common-mode transmission zeros using cross-shaped resonator," IEEE Microw. Wireless Compon. Lett., vol. 24, no. 12, pp. 854-856, Dec. 2014.

[30] W. Feng, W. Che, and Q. Xue, "High selectivity wideband differential bandpass filter with wideband common mode suppression using marchand balun," in Proc. IEEE Int. Wireless Symp., Xian, China, Mar. 2014, pp. 1-4.

[31] L. Li, J. Bao, J.-J. Du, and Y.-M. Wang, "Differential wideband bandpass filters with enhanced common-mode suppression using internal coupling technique," IEEE Microw. Wireless Compon. Lett., vol. 24, no. 5, pp. 300-302, May 2014.

[32] J. G. Zhou, Y.-C. Chiang, and W. Che, "Compact wideband balanced bandpass filter with high common-mode suppression based on cascade parallel coupled lines," IET Microw., Antennas Propag., vol. 8, no. 8, pp. 564-570, Jun. 2014.

[33] P. Vélez, J. Selga, M. Sans, J. Bonache, and F. Martin, "Design of differential-mode wideband bandpass filters with wide stop band and common-mode suppression by means of multisection mirrored stepped impedance resonators (SIRs)," in IEEE MTT-S Int. Microw. Symp. Dig., Phoenix, AZ, USA, May 2015, pp. 1-4.

[34] M. Sans et al., "Automated design of common-mode suppressed balanced wideband bandpass filters by means of aggressive space mapping," IEEE Trans. Microw. Theory Techn., vol. 63, no. 12, pp. 3896-3908, Dec. 2015.

[35] M. Sans et al., "Automated design of balanced wideband bandpass filters based on mirrored stepped impedance resonators (SIRs) and interdigital capacitors," Int. J. Microw. Wireless Technol., vol. 8, nos. 4-5, pp. 731-740, 2016.

[36] M. Sans et al., "Optimized wideband differential-mode bandpass filters with broad stopband and common-mode suppression based on multisection stepped impedance resonators and interdigital capacitors," in IEEE MTT-S Int. Microw. Symp. Dig., Seville, Spain, May 2017, pp. $10-12$.

[37] W. Feng and W. Che, "Novel wideband differential bandpass filters based on T-shaped structure," IEEE Trans. Microw. Theory Techn., vol. 60, no. 6, pp. 1560-1568, Jun. 2012.

[38] X.-H. Wu, Q.-X. Chu, and L.-L. Qiu, "Differential wideband bandpass filter with high-selectivity and common-mode suppression," IEEE Microw. Wireless Compon. Lett., vol. 2, no. 12, pp. 644-646, Dec. 2013.

[39] T. B. Lim and L. Zhu, "Differential-mode ultra-wideband bandpass filter on microstrip line," Electron. Lett., vol. 45, no. 22, pp. 1124-1125, 2009.

[40] X.-H. Wu and Q.-X. Chu, "Compact differential ultra-wideband bandpass filter with common-mode suppression," IEEE Microw. Wireless Compon. Lett., vol. 22, no. 9, pp. 456-458, Sep. 2012.

[41] A. M. Abbosh, "Ultrawideband balanced bandpass filter," IEEE Microw. Wireless Compon. Lett., vol. 21, no. 9, pp. 480-482, Sep. 2011.

[42] H. T. Zhu, W. J. Feng, W. Q. Che, and Q. Xue, "Ultra-wideband differential bandpass filter based on transversal signal-interference concept," Electron. Lett., vol. 47, no. 18, pp. 1033-1035, Sep. 2011.

[43] X.-H. Wang, H. Zhang, and B.-Z. Wang, "A novel ultra-wideband differential filter based on microstrip line structures," IEEE Microw. Wireless Compon. Lett., vol. 23, no. 3, pp. 128-130, Mar. 2013.

[44] S. Shi, W.-W. Choi, W. Che, K.-W. Tam, and Q. Xue, "Ultra-wideband differential bandpass filter with narrow notched band and improved common-mode suppression by DGS," IEEE Microw. Wireless Compon. Lett., vol. 22, no. 4, pp. 185-187, Apr. 2012.

[45] C.-H. Lee, C.-I. G. Hsu, and C.-J. Chen, "Band-notched balanced UWB BPF with stepped-impedance slotline multi-mode resonator," IEEE Microw. Wireless Compon. Lett., vol. 22, no. 4, pp. 182-184, Apr. 2012.

[46] J. Shi, C. Shao, J.-X. Chen, Q.-Y. Lu, Y. Peng, and Z.-H. Bao, "Compact low-loss wideband differential bandpass filter with high common-mode suppression," IEEE Microw. Wireless Compon. Lett., vol. 23, no. 9, pp. 480-482, Sep. 2013.

[47] P. Vélez et al., "Ultra-compact $\left(80 \mathrm{~mm}^{2}\right)$ differential-mode ultrawideband (UWB) bandpass filters with common-mode noise suppression," IEEE Trans. Microw. Theory Techn., vol. 63, no. 4, pp. 1272-1280, Apr. 2015.

[48] D. M. Pozar, Microwave Engineering, 4th ed. Hoboken, NJ, USA: Wiley, 2012.

[49] J.-S. Hong, Microstrip Filters for RF/Microwave Applications, Hoboken, NJ, USA: Wiley, 2001.
[50] J. W. Bandler, R. M. Biernacki, S. H. Chen, P. A. Grobelny, and R. H. Hemmers, "Space mapping technique for electromagnetic optimization," IEEE Trans. Microw. Theory Techn., vol. 42, no. 12, pp. 2536-2544, Dec. 1994.

[51] J. W. Bandler, R. M. Biernacki, S. H. Chen, R. H. Hemmers, and K. Madsen, "Electromagnetic optimization exploiting aggressive space mapping," IEEE Trans. Microw. Theory Techn., vol. 43, no. 12, pp. 2874-2882, Dec. 1995.

[52] S. Koziel, Q. S. Cheng, and J. W. Bandler, "Space mapping," IEEE Microw. Mag., vol. 9, no. 6, pp. 105-122, Dec. 2008.

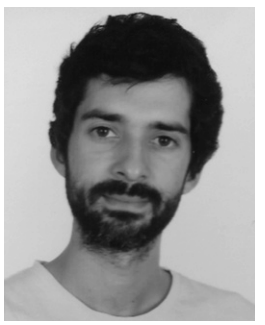

Marc Sans was born in Terrassa (Barcelona), Spain, in 1982. He received the B.S. degree in telecommunications engineering-electronic systems in 2006, the M.S. degree in telecommunications engineering in 2008, and the M.S. degree in electronics engineering in 2013 from the Universitat Autònoma de Barcelona (UAB), Barcelona.

In 2008 he started his professional career as an RF Engineer at Sony-FTVE in the RF group focused on the development of TV receivers. In 2010 he started working as an RF Design Engineer at Mier Comunicaciones S.A., where he was in charge of the design of passive and active devices for VHF-UHF broadcasting units. Since December 2015 up to the present, he is working as an Innovation RF Design Engineer at Ampleon. Meanwhile, in 2014 he joined the CIMITEC research group, mainly focused on metamaterial technology development, to start up his Ph.D. degree consisting in the synthesis of microwave devices based on EM optimization techniques.

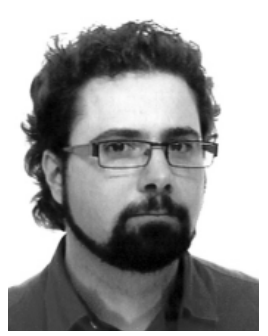

Jordi Selga (S'11-M'14) was born in Barcelona, Spain, in 1982. He received the B.S. degree in telecommunications engineering-electronic systems, M.S. degree in electronics engineering, and $\mathrm{Ph} . \mathrm{D}$. degree in electronics engineering from the Universitat Autònoma de Barcelona (UAB), Barcelona.

Since 2008, he has been a member of CIMITECUAB, Bellaterra, Spain. He is currently with $\mathrm{UAB}$, where he is involved in the development of metamaterials, CAD design of microwave devices, EM optimization methods, and automated synthesis of planar microwave components.

Dr. Selga is a Reviewer of IEEE MicrowaVE AND Wireless COMPONENTS LetTers, IET Microwave, Antennas and Propagation among others specialized journals in the RF/microwave field. He was a holder of a National Research Fellowship from the Formación de Profesorado Universitario Program of the Education and Science Ministry (Reference AP2008-4707).

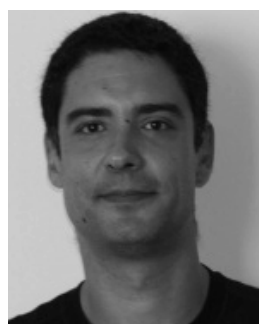

Paris Vélez (S'10-M'14) was born in Barcelona, Spain, in 1982. He received the degree in telecommunications engineering, with a specialization in electronics, Electronics Engineering degree, and $\mathrm{Ph} . \mathrm{D}$. degree in electrical engineering from the Universitat Autònoma de Barcelona, Barcelona, Spain, in 2008, 2010, and 2014, respectively. His Ph.D. thesis concerned common mode suppression differential microwave circuits based on metamaterial concepts and semilumped resonators.

$\mathrm{He}$ is currently with the LAAS-CNRS, Toulouse, France, where he is involved in metamaterials sensors for fluidics detection. His current research interests include the miniaturization of passive circuits $\mathrm{RF} / \mathrm{microwave}$ and sensor-based metamaterials.

Dr. Vélez was a recipient of a Predoctoral Teaching and Research Fellowship by the Spanish Government from 2011 to 2014. He was the recipient of a TECNIOSpring Fellowship cofounded by the Mari Curie Program. He is a Reviewer for the IEEE TRANSACTIONS ON MICROWAVE THEORY AND TECHNIQUES and other journals. 


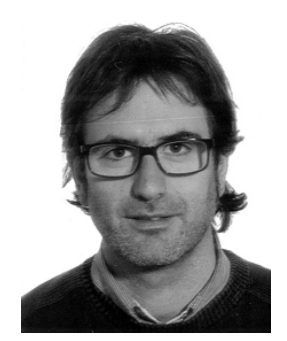

Jordi Bonache (S'05-M'07) was born in Barcelona, Spain, in 1976. He received the Physics and Electronics Engineering degrees and Ph.D. degree in electronics engineering from the Universitat Autònoma de Barcelona, Barcelona, in 1999 and 2001 and 2007, respectively.

In 2000, he joined the High Energy Physics Institute, Barcelona, where he was involved in the design and implementation of the control and monitoring system of the MAGIC telescope. In 2001, he joined the Department of Electronics Engineering, Universitat Autònoma de Barcelona, where he is currently an Associate Professor. From 2006 to 2009, he was an Executive Manager with CIMITEC, Bellaterra, Spain, where he led research on RFID and antennas. His current research interests include active and passive microwave devices, metamaterials, antennas, and RFID.

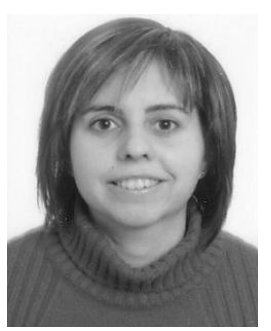

Ana Rodríguez (GS'09-M'12) was born in Lugo, Spain. She received the Telecommunications Engineering degree from the Universidade de Vigo, Vigo, Spain, in 2008, and the Master en Tecnología, Sistemas y Redes de Comunicaciones and the $\mathrm{Ph} . \mathrm{D}$. degree from the Universitat Politècnica de València (UPV), València, Spain, in 2010 and 2014, respectively.

She participated in the Erasmus Exchange Program, developing her master's thesis at the University of Oulu, Oulu, Finland. Since 2008, she has been with the Institute of Telecommunications and Multimedia Applications, UPV. Her current research interests include the CAD design of microwave devices, EM optimization methods, and metamaterials.

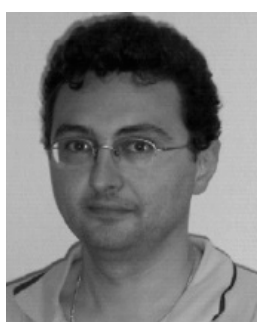

Vicente E. Boria (S'91-A'99-SM'02) was born in Valencia, Spain, in 1970. He received the Ingeniero de Telecomunicación degree (with First-Class Hons.) and Doctor Ingeniero de Telecomunicación degree from the Universidad Politécnica de Valencia, Valencia, in 1993, and 1997, respectively.

In 1993, he joined the Departamento de Comunicaciones, Universidad Politécnica de Valencia, where he has been a Full Professor since 2003. From 1995 to 1996, he was a Spanish Trainee with the European Space Research and Technology Centre, European Space Agency, Noordwijk, The Netherlands, where he was involved in the EM analysis and design of passive waveguide devices. He has authored or co-authored 10 chapters in technical textbooks, 135 papers in refereed international technical journals, and over 180 papers in international conference proceedings. His current research interests include the analysis and automated design of passive components, left-handed and periodic structures, as well as on the simulation and measurement of power effects in passive waveguide systems.

Dr. Boria has been a member of the IEEE Microwave Theory and Techniques Society (IEEE MTT-S) and the IEEE Antennas and Propagation Society since 1992. He is also a member of the Technical Committees of the IEEE-MTT International Microwave Symposium and the European Microwave Conference. He is a Reviewer for the IEEE TRANSACTIONS on Microwave Theory and Techniques, and Proceeding of the IET (Microwaves, Antennas and Propagation). He currently serves as an Associate Editor for IEEE MICROWAVE AND WIRELESS COMPONENTS LETTERS and IET Electronics Letters.

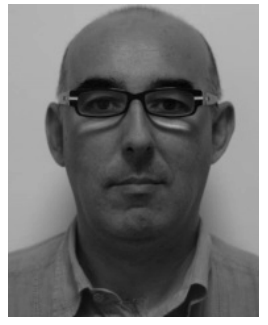

Ferran Martín (M'04-SM'08-F'12) was born in Barakaldo (Vizcaya), Spain, in 1965. He received the B.S. degree in physics from the Universitat Autònoma de Barcelona (UAB), Barcelona, Spain, in 1988, and the Ph.D. degree in 1992.

From 1994 to 2006, he was an Associate Professor of electronics with the Departament d'Enginyeria Electrònica, UAB, where he has been a Full Professor of electronics since 2007. He is currently the Head of the Microwave Engineering, Metamaterials and Antennas Group, UAB, and the Director of the CIMITEC. He has been involved in different research activities including modelling and simulation of electron devices for high-frequency applications, millimeter-wave and terahertz generation systems, and the application of electromagnetic bandgaps to microwave and millimeter-wave circuits, metamaterials and their application to the miniaturization and optimization of microwave circuits and antennas. He has authored or co-authored over 500 technical conference, letter, journal papers, and book chapters. He coauthored the book on metamaterials Metamaterials With Negative Parameters: Theory, Design and Microwave Applications (Wiley, 2008), authored Artificial Transmission Lines for RF and Microwave Applications (Wiley, 2015), and he has generated $17 \mathrm{Ph} . \mathrm{Ds}$. He has filed several patents on metamaterials and has headed several development contracts.

Prof. Martín is a member of the IEEE Microwave Theory and Techniques Society (IEEE MTT-S). He is also a member of the Technical Committees of the European Microwave Conference and the International Congress on Advanced Electromagnetic Materials in Microwaves and Optics (Metamaterials). He has been a Fellow of the IET since 2016. He was a recipient of the 2006 Duran Farell Prize for Technological Research. He holds the Parc de Recerca UAB-Santander Technology Transfer Chair and two ICREA ACADEMIA Awards (2008 and 2013). He is a Reviewer for the IEEE TRANS ACTIONS ON MiCROWAVE THEORY AND TECHNIQUES and IEEE MICROWAVE AND WIRELESS COMPONENTS LETTERS, among many other journals, and he serves as a member of the Editorial Board of IET Microwaves, Antennas and Propagation and the International Journal of RF and Microwave Computer-Aided Engineering. He served as a Guest Editor for three special issues on metamaterials in three international journals. $\mathrm{He}$ has organized several international events related to metamaterials, including workshops at the IEEE MTT-S International Microwave Symposium in 2005 and 2007, the European Microwave Conference in 2009, and the Fifth International Congress on Advanced Electromagnetic Materials in Microwaves and Optics (Metamaterials 2011), where he was the Chair of the Local Organizing Committee. 